Chapter 12

\title{
Application of Nanocomposites for Supercapacitors: Characteristics and Properties
}

\author{
Dongfang Yang \\ Additional information is available at the end of the chapter
}

http://dx.doi.org/10.5772/50409

\section{Introduction}

Supercapacitors, ultracapacitors or electrochemical capacitors (ECs), are energy storage devices that store energy as charge on the electrode surface or sub-surface layer, rather than in the bulk material as in batteries, therefore, they can provide high power due to their ability to release energy more easily from surface or sub-surface layer than from the bulk. Since charging-discharging occurred on the surface, which does not induce drastic structural changes upon electroactive materials, supercapacitors possess excellent cycling ability. Due to those unique features, supercapacitors are regarded as one of the most promising energy storage devices. There are two types of supercapacitors: electrochemical double layer capacitors (EDLCs) and pseudocapacitors. In EDLCs, the energy is stored electrostatically at the electrode-electrolyte interface in the double layer, while in pseudocapacitors charge storage occurs via fast redox reactions on the electrode surface. There are three major types of electrode materials for supercapacitors: carbon-based materials, metal oxides/hydroxides and conducting polymers. Carbon-based materials such as activated carbon, mesoporous carbon, carbon nanotubes, graphene and carbon fibres are used as electrode active materials in EDLCs, while conducting polymers such as polyaniline, polypyrrole and polythiophene or metal oxides such as $\mathrm{MnO}_{2}, \mathrm{~V}_{2} \mathrm{O}_{5}$, and $\mathrm{RuO}_{2}$ are used for pseudocapacitors. EDLCs depends only on the surface area of the carbon-based materials to storage charge, therefore, often exhibit very higher power output and better cycling ability. However, EDLCs have lower energy density values than pseudocapacitors since pseudocapacitors involve redox active materials to store charge both on the surface as well as in sub-surface layer.

Although carbon-based materials, metal oxides/hydroxides and conducting polymers are the most common electroactive materials for supercapacitor, each type of material has its own unique advantages and disadvantages, for example, carbon-based materials can provide high 
power density and long life cycle but its small specific capacitance (mainly double layer capacitance) limits its application for high energy density devices. Metal oxides/hydroxides possess pseudocapacitance in additional to double layer capacitance and have wide charge/discharge potential range; however, they have relatively small surface area and poor cycle life. Conducting polymers have the advantages of high capacitance, good conductivity, low cost and ease of fabrication but they have relatively low mechanical stability and cycle life. Coupling the unique advantages of these nano-scale dissimilar capacitive materials to form nanocomposite electroactive materials is an important approach to control, develop and optimize the structures and properties of electrode material for enhancing their performance for supercapacitors. The properties of nanocomposite electrodes depend not only upon the individual components used but also on the morphology and the interfacial characteristics. Recently, considerable efforts have been placed to develop all kinds of nanocomposite capacitive materials, such as mixed metal oxides, conducting polymers mixed with metal oxides, carbon nanotubes mixed with conducting polymers, or metal oxides, and graphene mixed with metal oxides or conducting polymers. Design and fabrication of nanocomposite electroactive materials for supercapacitors applications needs the consideration of many factors, such as material selection, synthesis methods, fabrication process parameters, interfacial characteristics, electrical conductivity, nanocrystallite size, and surface area, etc. Although significant progress has been made to develop nanocomposite electroactive materials for supercapacitor applications, there are still a lot of challenges to be overcome. This chapter will summarize the most recent development of this new area of research including the synthesis methods currently used for preparing nanocomposite electroactive materials, types of nanocomposite electroactive materials investigated,structural and electrochemical characterization of nanocomposites, unique capacitive properties of nanocomposite materials, and performance enhancement of nanocomposite electroactive materials and its mechanism.

\section{Fabrication and characterization of nanocomposite active electrode materials}

\subsection{Fabrication methods}

To prepare mixed metal oxide nanocomposites, various synthesis methods including solid state reactions (i.e. thermal decomposition of mechanical mixtures of metal salts), mechanical mixing of metal oxides (i.e. ball milling), and chemical co-precipitation and electrochemical anodic deposition from solutions containing metal salts, have been used. For example (examples in section 2.1 will be described in more details in section 3 and the references will also be given in section 3), $\mathrm{Mn}-\mathrm{Pb}$ and $\mathrm{Mn}-\mathrm{Ni}$ mixed oxide nanocomposites were prepared by reduction of $\mathrm{KMnO}_{4}$ with $\mathrm{Pb}(\mathrm{II})$, and $\mathrm{Ni}(\mathrm{II})$ salts to form amorphous mixed oxide precipitant. Mn-V-W oxide, and Mn-V-Fe oxide were then directly deposited by anodic deposition on conductive substrates from aqueous solution consisting of mixed metal salts. Directly anodizing Ti-V alloys in ethylene glycol with HF electrolyte was used to synthesis mixed 
$\mathrm{V}_{2} \mathrm{O}_{5}-\mathrm{TiO}_{2}$ nanotube arrays. Hydrothermal process was also used to prepare $\mathrm{SnO}_{2}-\mathrm{Al}_{2} \mathrm{O}_{3}$ mixed oxide nanocomposites involving urea as the hydrolytic agent in an autoclave.

Carbon nanotubes (CNTs)-metal oxide nanocomposites were prepared by either mechanically mixing CNTs with metal oxides in a mortar, or depositing metal oxides directly on CNTs by metal-organic chemical vapour deposition (CVD), wet-chemical precipitation, or electrochemically deposition. For example, $\mathrm{IrO}_{2}$ nanotubes were deposited on multiwall CNTs using metal-organic CVD with the iridium source of $\left(\mathrm{C}_{6} \mathrm{H}_{7}\right)\left(\mathrm{C}_{8} \mathrm{H}_{12}\right) \mathrm{Ir}$ at $350 \circ \mathrm{C}$ to form $\mathrm{IrO}_{2}-\mathrm{CNTS}$ nanocomposite. The CNTs themselves were initially grown on stainless steel plate using thermal CVD. The $\mathrm{MnO}_{2}-\mathrm{CNTs}$ nanocomposites were synthesized by direct current anodic deposition of $\mathrm{MnO}_{2}$ from the $\mathrm{MnSO}_{4}$ solution over electrophoretically deposited CNTs on the Ni substrate. $\mathrm{RuO}_{2}$-CNTs was formed by impregnating CNTs with a ruthenium nitrosylnitrate solution and then followed by heat treatment to form composite electrode.

Nanocomposite of a conducting polymer with metal oxide, CNTs or graphene (GN) were mainly synthesized by in situ polymerization in solutions containing monomers of the conducting polymer and suspension of CNTs, metal oxide nanoparticles or GN nanosheets. For example, CNTs- polyaniline (PANI) nanocomposite was prepared from a solution consisting of CNTs and aniline monomer. With addition of an oxidant solution containing $\left(\mathrm{NH}_{4}\right)_{2} \mathrm{~S}_{2} \mathrm{O}_{8}$, polymerization of aniline on the surface of CNTs occurred to form CNTs-PANI nanocomposite. $\mathrm{MoO}_{3}$-Poly 3,4-ethylenedioxythiophene (PEDOT) nanocomposites was synthesized by adding 3,4-ethylenedioxythiophene monomer into a lithium molybdenum nanoparticle suspension, and subsequently, Iron (III) chloride $\left(\mathrm{FeCl}_{3}\right)$ was added to the suspension as the oxidizing agent under microwave hydrothermal conditions for polymerization to occur. GN-PEDOT nanocomposite was chemically synthesized by oxidative polymerization of ethylene dioxythiophene using ammonium peroxydisulfate $\left.\left[\left(\mathrm{NH}_{4}\right)_{2} \mathrm{~S}_{2} \mathrm{O}_{8}\right)\right]$ and $\mathrm{FeCl}_{3}$ as oxidizing agents in a solution containing sodium polystyrene sulfonate $\mathrm{Na}$ salt, $\mathrm{HCl}$, EDOT monomer and GN. G-PANI nanocomposite was chemically synthesized by oxidative polymerization of aniline monomers using ammonium peroxydisulfate $\left.\left[\left(\mathrm{NH}_{4}\right)_{2} \mathrm{~S}_{2} \mathrm{O}_{8}\right)\right]$ in solution containing GN.

GN-metal oxide nanocomposites were prepared by chemical precipitation of metal oxide in the presence of GN nanosheets in the solution. For example, $\mathrm{GN}-\mathrm{CeO}_{2}$ nanocomposite was prepared by adding $\mathrm{KOH}$ solution dropwise into a $\mathrm{Ce}\left(\mathrm{NO}_{3}\right)_{3}$ aqueous solution in the presence of 3D GN material, followed by filtering, and drying. The $\mathrm{GN}-\mathrm{SnO}_{2} / \mathrm{CNTs}$ nanocomposite was synthesized by ultrasonicating the mixture of chemically functionalized GN and $\mathrm{SnO}_{2}-\mathrm{CNTs}$ in water. Normally, GN sheets were synthesized via exfoliation of graphite oxide in hydrogen environment at low temperature while graphite oxide (GO) was prepared normally by Hummers method.

\subsection{Structure, electrical, chemical composition and surface area characterization}

X-ray diffraction (XRD), scanning electron microscopy/energy-dispersive analysis (SEM/ EDX), high-resolution transmission electron microscopy (HRTEM), infrared spectra (IR) and the Brunauer-Emmett-Teller (BET) specific surface areas were the most common analytical techniques to characterize the morphologies, structures, chemical composition and surface 
area of nanocomposite electroactive materials. XRD analysis was carried out for the nanocomposite samples containing metal oxides to examine the crystallinity and crystal phases of the oxide materials. IR spectra were used for the identification of the characteristic bands of a polymer for nanocomposites consisting of conducting polymers. SEM was used for morphological analysis of nanocomposites, while EDX was used to determine their chemical composition. The electrical conductivity of nanocomposites was obtained normally using a four-probe technique. To do the measurement, the nanocomposite samples were ground into fine powders and then were pressed as pellets. The weight loss of nanocomposite material and the heat flow associated with the thermal decomposition during synthesis or heat treatment were studied by thermogravimetric analysis (TGA) and differential thermal analysis (DTA).

\subsection{Electrochemical characterizations}

Cyclic voltammetry (CV) was usually conducted to characterize the nanocomposite electrode in a three-electrode cell in either aqueous electrolytes or organic electrolytes using an electrochemistry workstation. The working electrode was metal plate or mesh (e.g. nickel, aluminium, stainless steel) coated with a mixture of nanocomposite and conductive carbon such as acetylene black with a binder such as PTFE or polyvinylidenedifluoride (PVDF). The reference electrodes were either saturated calomel electrode (SCE), $\mathrm{Ag} / \mathrm{AgCl}$ or others. The counter electrode was typically platinum foil. The specific current and specific capacitance of nanocomposite was determined by the CV current value, scan rate and the weight of nanocomposites.

Galvanostatic charge-discharge cycling was performed with two electrode system having identical electrodes made of same nanocomposite electroactive material. Constant current densities ranging from 0.5 to $10 \mathrm{~mA} / \mathrm{cm}^{2}$ were typically employed for charging/discharging the cell in the voltage range $0-1 \mathrm{~V}$ for aqueous electrolytes or $0-2.7 \mathrm{~V}$ for organic electrolytes. The discharge capacitance $(\mathrm{C})$ is estimated from the slope $(\mathrm{dV} / \mathrm{dt})$ of the linear portion of the discharge curve using the expression.

$$
\mathrm{C}=\mathrm{IdV} / \mathrm{dt}
$$

The weight of the active material of the two electrodes is same in a symmetric supercapacitor. The specific capacitance (Cs) of the single electrode can thus be expressed as:

$$
\mathrm{C}_{\mathrm{s}}=2 \mathrm{C} / \mathrm{m}
$$

where $m$ is the active material mass of the single electrode. The energy density $\left(E_{d}\right)$ of the capacitor can be expressed as,

$$
\mathrm{E}_{\mathrm{d}}=1 / 2\left(\mathrm{C}_{\mathrm{s}} \mathrm{V}_{\text {max }}^{2}\right)
$$

The coulomb efficiency ' $\eta$ ' was evaluated using the following relation, 


$$
h=t_{d} / t_{c} \times 100 \%
$$

where $t_{c}$ and $t_{d}$ are the time of charge and discharge respectively.

Experiments of electrochemical impedance spectra (EIS) were also performed with a two electrode system having identical electrodes made of same nanocomposite active electrode materials at open circuit potential (OCP) over the frequency range $10 \mathrm{kHz}-10 \mathrm{mHz}$ with a potential amplitude normally of $5 \mathrm{mV}$. The impedance spectra usually show a single semicircle in the high frequency region and nearly vertical line in the low frequency region for a supercapacitor, which indicates that the electrode process is controlled by electrochemical reaction at high frequencies and by mass transfer at low frequencies. The intercept of the semi-circle with real axis $\left(Z_{\text {real }}\right)$ at high frequencies is the measure of internal resistance $\left(R_{s}\right)$ which may be due to (i) ionic resistance of the solution or electrolyte, (ii) intrinsic resistance of the active electrode materials and (iii) interfacial resistance between the electrode and current collector. The origin of the semi-circle at higher frequency range is due to ionic charge transfer resistance $\left(R_{c t}\right)$ at the electrode-electrolyte interface. The diameter of the semi-circle along the real axis $\left(Z_{\text {dia }}\right)$ gives the charge transfer resistance $R_{c t}$.

\section{Performance of various types of nanocomposite active electrode materials}

\subsection{Mixed pseudocapacitive metal oxide nanocomposites}

Metal oxides like such as $\mathrm{RuO}_{2}, \mathrm{MnO}_{2}, \mathrm{Co}_{3} \mathrm{O}_{4}, \mathrm{NiO}, \mathrm{SnO}_{2}, \mathrm{Fe}_{3} \mathrm{O}_{4}$, and $\mathrm{V}_{2} \mathrm{O}_{5}$ have been employed as electroactive materials for pseudocapacitors. Those metal oxides typically have several redox states or structures and contribute to the charge storage in pseudocapacitors via fast redox reactions. The remarkable performance of $\mathrm{RuO}_{2}$ in supercapacitors (exhibits the highest specific capacitance values of $720 \mathrm{~F} \mathrm{~g}^{-1}$ ) has stimulated many interests in investigating metal oxide system for supercapacitor applications. The commercial use of $\mathrm{RuO}_{2}$, however, is limited owing to its high cost and toxic nature. Other simple metal oxides usually have some limitations such as poor electrical conduction, insufficient electrochemical cycling stability, limited voltage operating window and low specific capacitance. Those limitations need to be addressed in order for commercial applications of supercapacitors based on metal oxides. Mixed binary or ternary metal oxides systems, such as Ni-Mn oxide, Mn-Co oxide, Mn-Fe oxide, Ni-Ti oxide, $\mathrm{Sn}-\mathrm{Al}$ oxide, $\mathrm{Mn}-\mathrm{Ni}-\mathrm{Co}$ oxide, $\mathrm{Co}-\mathrm{Ni}-\mathrm{Cu}$ oxide and $\mathrm{Mn}-\mathrm{Ni}-\mathrm{Cu}$ oxide have shown improved properties as electroactive materials for pseudocapacitors and have shed new lights in this area of research. The following section summarizes the recent development in seeking electroactive mixed metal oxide nanocomposites for pseudocapacitors. 


\subsubsection{Mixed manganese oxides}

The natural abundance and low cost of Mn oxides, along with their satisfactory energy-storage performance in mild electrolytes and environmental compatibility, has made them the most promising new electroactive material for the pseudocapacitor applications. However, Mn oxides has limitations such as low surface areas, poor electrical conductivity and relatively small specific capacitance value. To improve the electrochemical performance of $\mathrm{Mn}$ oxides for pseudocapacitors, many efforts have been devoted to incorporate various transition metals into Mn oxides to form mixed metal oxide nanocomposites with controlled micro-/nanostructures in order to improve their electrochemical characteristics. The understanding of their synergistic effect and the eventual design of an integrated material architecture in which each component's properties can be optimized and a fast ion and electron transfer will be guaranteed still remains a great challenge.

Jiang et al. [1] designed and synthesized $\mathrm{MnO}_{2}$ nanoflakes- $\mathrm{Ni}(\mathrm{OH})_{2}$ nanowires composites that can be used in both neutral and alkaline electrolytes and have very high cycling stability. The nanocomposites with $70.4 \mathrm{wt} . \% \mathrm{MnO}_{2}$ content exhibited specific capacitance of $355 \mathrm{~F} \mathrm{~g}^{-1}$ with excellent cycling stability ( $97.1 \%$ retention after 3000 cycles) in $1 \mathrm{M} \mathrm{Na}_{2} \mathrm{SO}_{4}$ neutral aqueous solution. In $1 \mathrm{M} \mathrm{KOH}$ aqueous alkaline solution, the $\mathrm{MnO}_{2}-\mathrm{Ni}(\mathrm{OH})_{2}$ nanocomposite with 35.5 wt. $\% \mathrm{MnO}_{2}$ content possessed a specific capacitance of $487.4 \mathrm{~F} \mathrm{~g}^{-1}$ also with excellent cycling stability. Such excellent capacitive behaviours are attributed by the authors to the unique $\mathrm{MnO}_{2}-$ $\mathrm{Ni}(\mathrm{OH})_{2}$ core-shell nanostructures as depicting in Figure $1(\mathrm{~b})$. The interconnected $\mathrm{MnO}_{2}$ nanoflakes were well-dispersed on the surface of $\mathrm{Ni}(\mathrm{OH})_{2}$ nanowires that creates highly porous surface morphology. This integrated structure can provide high surface area and more active sites for the redox reactions. The specific capacitance and Coulombic efficiency as function of cycle number at a current density of $10 \mathrm{~A} \mathrm{~g}^{-1}$ for up to 3000 cycles is also shown in Figure 1(a) for the $\mathrm{MnO}_{2}-\mathrm{Ni}(\mathrm{OH})_{2}$ nanocomposite. After long cycling, the $\mathrm{Ni}(\mathrm{OH})_{2}-\mathrm{MnO}_{2}$ nanocomposites are overall preserved with little structural deformation, as shown in Figure 1(c) and (d). Oxides of $\mathrm{Pb}, \mathrm{Fe}, \mathrm{Mo}$, and $\mathrm{Co}$ were also incorporated into $\mathrm{MnO}_{2}$ to form mixed metal oxide nanocomposites. Kim et al.[2] synthesized mixed oxides of $\mathrm{Mn}$ with $\mathrm{Pb}$ or $\mathrm{Ni}$ by reduction of $\mathrm{KMnO}_{4}$ with either lead(II) acetate-manganese acetate or nickel(II) acetate-manganese acetate reducing solutions. Characterization of the nanocomposite electrodes were carried out using cyclic voltammetry, galvanostatic charge-discharge, XRD, BET analysis, and TGA. The results showed that by introducing $\mathrm{Ni}$ and $\mathrm{Pb}$ into $\mathrm{MnO}_{2}$, the surface area of the mixed oxide increased due to the formation of micropores. The specific capacitance increased from $166 \mathrm{~F} \mathrm{~g}^{-1}$ (for $\mathrm{MnO}_{2}$ ) to 210 and $185 \mathrm{~F} \mathrm{~g}^{-1}$ for $\mathrm{Mn}-\mathrm{Ni}$ and $\mathrm{Mn}-\mathrm{Pb}$ mixed oxides, respectively. Kim et al. [2] also found that annealing of the nanocomposites can affect their capacitance: transition from amorphous to a crystalline structure occurred at high temperature $\left(400^{\circ} \mathrm{C}\right)$ reduces the specific capacitance. Binary $\mathrm{Mn}-\mathrm{Fe}$ oxide was electroplated on graphite substrates by Lee et al. [3] at a constant applied potential of $0.8 \mathrm{~V}$ vs. SCE in a mixed plating solution of $\mathrm{Mn}\left(\mathrm{CH}_{3} \mathrm{COO}\right)_{2}$ and $\mathrm{FeCl}_{3}$. The electrochemical behaviours of the as-deposited and the annealed mixed oxide nanocomposites were characterized by cyclic voltammetry in $2 \mathrm{M} \mathrm{KC1}$ solution. Lee et al. found that as-deposited $\mathrm{Mn}-\mathrm{Fe}$ binary oxide has porous structure and is amorphous. After annealing at $100^{\circ} \mathrm{C}$ to remove the adsorbed water, the partially hydrous mixed oxide has optimized ionic and 
electronic conductivity and gives rise to the best pseudocapacitive performance. However, if the annealing temperature is increased to higher, the mixed oxide loses it porosity and slowly crystalizes which leads to the decrease in specific capacitance. A series of Mn and Mo mixed oxides (i.e. Mn-Mo-X (X=W, Fe, Co)) were investigated by Ye et al.[4] and they found that the specific capacitance of Mo doped Mn oxides are higher than that of pure Mn oxide. The MnMo-Fe oxide reach a high specific capacitance value of $278 \mathrm{~F} \mathrm{~g}^{-1}$ in aqueous $0.1 \mathrm{M} \mathrm{Na}_{2} \mathrm{SO}_{4}$ electrolyte at a scan rate of $20 \mathrm{mV} \mathrm{s}^{-1}$ and has a rectangular-shaped voltammogram. The improvement in capacitance of Mn oxides doped with molybdenum was attributed by the authors to the formation of nanostructure and the existence of low crystallinity. The above results show that mixed metal oxides with amorphous structure have better specific capacitance than that of crystalline structure. Incorporation of various transition metals into Mn oxides creates more porous structures, therefore increase their specific capacitance.
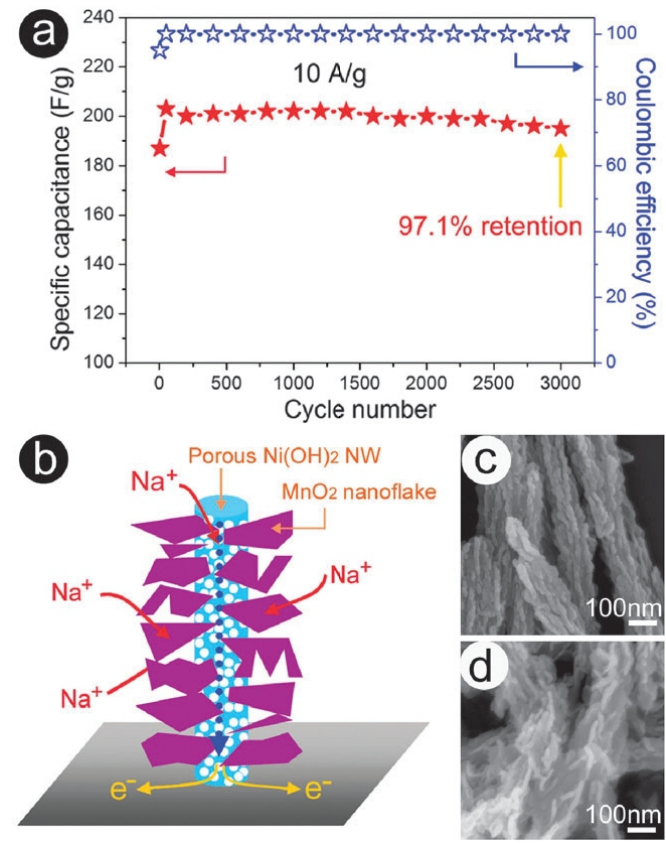

Figure 1. a) Specific capacitance as a function of cycle number at $10 \mathrm{~A} \mathrm{~g}^{-1}$, (b) schematic of the charge storage advantage of the $\mathrm{Ni}(\mathrm{OH})_{2}-\mathrm{MnO}_{2}$ core-shell nanowires, (c) and (d) SEM images of the $\mathrm{Ni}(\mathrm{OH})_{2}-\mathrm{MnO}_{2}$ core-shell nanowires before and after 3000 cycles (from ref. 1)

Advance thin film physical vapour deposition methods were also used to prepare mixed metal oxide nanocomposite for supercapacitor electroactive materials research. Thin films of manganese oxide doped with various percentages of cobalt oxide were grown by pulsed laser deposition (PLD) on silicon wafers and stainless steel substrates at our laboratory [5]. Before investigated Co-doped manganese oxide film, our team [6] developed different PLD 
processing parameters (i.e. temperature, oxygen pressure) to produce various chemical compositions and phases of manganese oxides such as pure crystalline phases of $\mathrm{Mn}_{2} \mathrm{O}_{3}$ and $\mathrm{Mn}_{3} \mathrm{O}_{4}$ as well as amorphous phase of $\mathrm{MnO}_{x}$. He then evaluated the pseudo-capacitance behaviours of these different phases of manganese oxides and found that the crystalline $\mathrm{Mn}_{2} \mathrm{O}_{3}$ phase has the highest specific current and capacitance, while the values for crystalline $\mathrm{Mn}_{3} \mathrm{O}_{4}$ films are the lowest. The specific current and capacitance values of the amorphous $\mathrm{MnO}_{x}$ films are in between $\mathrm{Mn}_{2} \mathrm{O}_{3}$ and $\mathrm{Mn}_{3} \mathrm{O}_{4}$. The specific capacitance of $\mathrm{Mn}_{2} \mathrm{O}_{3}$ films of 120 $\mathrm{nm}$ thick reaches $210 \mathrm{~F} \mathrm{~g}^{-1}$ at $1 \mathrm{mV} \mathrm{s}^{-1}$ scan rate with excellent stability and cyclic durability. He then doped amorphous $\mathrm{MnO}_{x}$ and crystalline $\mathrm{Mn}_{2} \mathrm{O}_{3}$ phases with $\mathrm{Co}_{3} \mathrm{O}_{4}$ and characterized the mixed Co-Mn oxide films with X-ray diffraction and CVs. The CVs recorded at a 20 $\mathrm{mV} \mathrm{s}^{-1}$ scan rate for un-doped and Co-doped amorphous $\mathrm{MnO}_{\mathrm{x}}$ films are shown in Figure 2(a), and their specific capacitance determined from the CV curves at scan rates of 5, 10, 20 and $50 \mathrm{mV} \mathrm{s}^{-1}$ are shown in Figure 2(b). The CVs in Figure 2 shows that the Co-doped amorphous $\mathrm{MnO}_{\mathrm{x}}$ films have larger specific currents and capacitances than the un-doped amorphous $\mathrm{MnO}_{\mathrm{x}}$ film. Low cobalt doping (3.0 atm.\%) had the greatest increase in capacitance, followed by $9.3 \mathrm{~atm} . \%$ cobalt doping. The $22.6 \mathrm{~atm} . \%$ cobalt doping had the least increase in specific capacitance. The operating potential window (between $\mathrm{H}_{2}$ evolution and $\mathrm{O}_{2}$ evolution due to decomposition of water) was shifted about $100 \mathrm{mV}$ toward more negative potentials for all the Co-Mn mixed oxide films. At a $5 \mathrm{mV} / \mathrm{s}$ scan rate, the $3.0 \mathrm{~atm}$ \% Co-doped $\mathrm{MnOx}$ film reached $99 \mathrm{~F} \mathrm{~g}^{-1}$, which is more than double that the $47 \mathrm{~F} \mathrm{~g}^{-1}$ observed for the undoped $\mathrm{MnO}_{\mathrm{x}}$ film. This result indicates that Co doping significantly improves the pseudocapacitance performance of amorphous manganese oxide. However, Co-doped crystalline $\mathrm{Mn}_{2} \mathrm{O}_{3}$ films did not show an improvement in specific current and capacitance compared with un-doped $\mathrm{Mn}_{2} \mathrm{O}_{3}$ crystalline films. High Co doping level (20.7 atm.\% doped) in the crystalline $\mathrm{Mn}_{2} \mathrm{O}_{3}$ films actually decreased both the specific current and capacitance values. These findings demonstrate that elemental doping is an effective way to alter the performance of pseudo-capacitive metal oxides. Our work also demonstrated that thin film deposition techniques such as PLD are very promising techniques for screening high performance mixed oxide active materials for supercapacitor applications.

\subsubsection{Other mixed metal oxides}

In addition to mixed manganese oxides, many other mixed metal oxides have also been investigated as electroactive materials for supercapacitor applications. $\mathrm{Co}_{3} \mathrm{O}_{4}-\mathrm{Ni}(\mathrm{OH})_{2}$ nanocomposites were synthesized by electrochemical deposition on the Ti substrate in a solution of $\mathrm{Ni}\left(\mathrm{NO}_{3}\right)_{2}, \mathrm{Co}\left(\mathrm{NO}_{3}\right)_{2}$ and $\mathrm{NH}_{4} \mathrm{Cl}$, then follows by heat treatment at $200^{\circ} \mathrm{C}$ [7]. The $\mathrm{CO}_{3} \mathrm{O}_{4^{-}}$ $\mathrm{Ni}(\mathrm{OH})_{2}$ electrodes exhibited high specific capacitance value of $1144 \mathrm{~F} \mathrm{~g}^{-1}$ at $5 \mathrm{mV} \mathrm{s}^{-1}$ and long-term cycliability. The excellent capacitive behaviours of $\mathrm{Co}_{3} \mathrm{O}_{4}-\mathrm{Ni}(\mathrm{OH})_{2}$ nanocomposite was attributed by the authors to the porous network structures that favour electron and ions transportation as well as faradic redox reactions of both couples of $\mathrm{Co}^{2+} / \mathrm{Co}^{3+}$ and $\mathrm{Ni}^{2+} / \mathrm{Ni}^{3+}$. Y. Yang et al. [8] prepared mixed $\mathrm{V}_{2} \mathrm{O}_{5}-\mathrm{TiO}_{2}$ nanotube arrays by anodizing $\mathrm{Ti}-\mathrm{V}$ alloys with different $\mathrm{V}$ compositions using an ethylene glycol with $0.2 \mathrm{M} \mathrm{HF}$ as the electrolyte at a comparably high anodization voltage. Well-defined nanotube structures were grown for alloys with vanadium content up to 18 at $\%$. The mixed $\mathrm{V}_{2} \mathrm{O}_{5}-\mathrm{TiO}_{2}$ nanotube arrays were found to 
exhibit greatly enhanced capacitive properties compared with pure $\mathrm{TiO}_{2}$ nanotubes. The specific capacitance of the mixed $\mathrm{V}_{2} \mathrm{O}_{5}-\mathrm{TiO}_{2}$ nanotubes can reach up to $220 \mathrm{~F} \mathrm{~g}^{-1}$ with an energy density of $19.56 \mathrm{Wh} \mathrm{kg}^{-1}$ and was found to be very stable in repeated cycles. Another interesting mixed oxide is $\mathrm{SnO}_{2}-\mathrm{Al}_{2} \mathrm{O}_{3}$ mixed oxide [9], which shows much greater electrochemical capacitance than pure $\mathrm{SnO}_{2}$ and was electrochemically and chemically stable even after cycling1000 times.
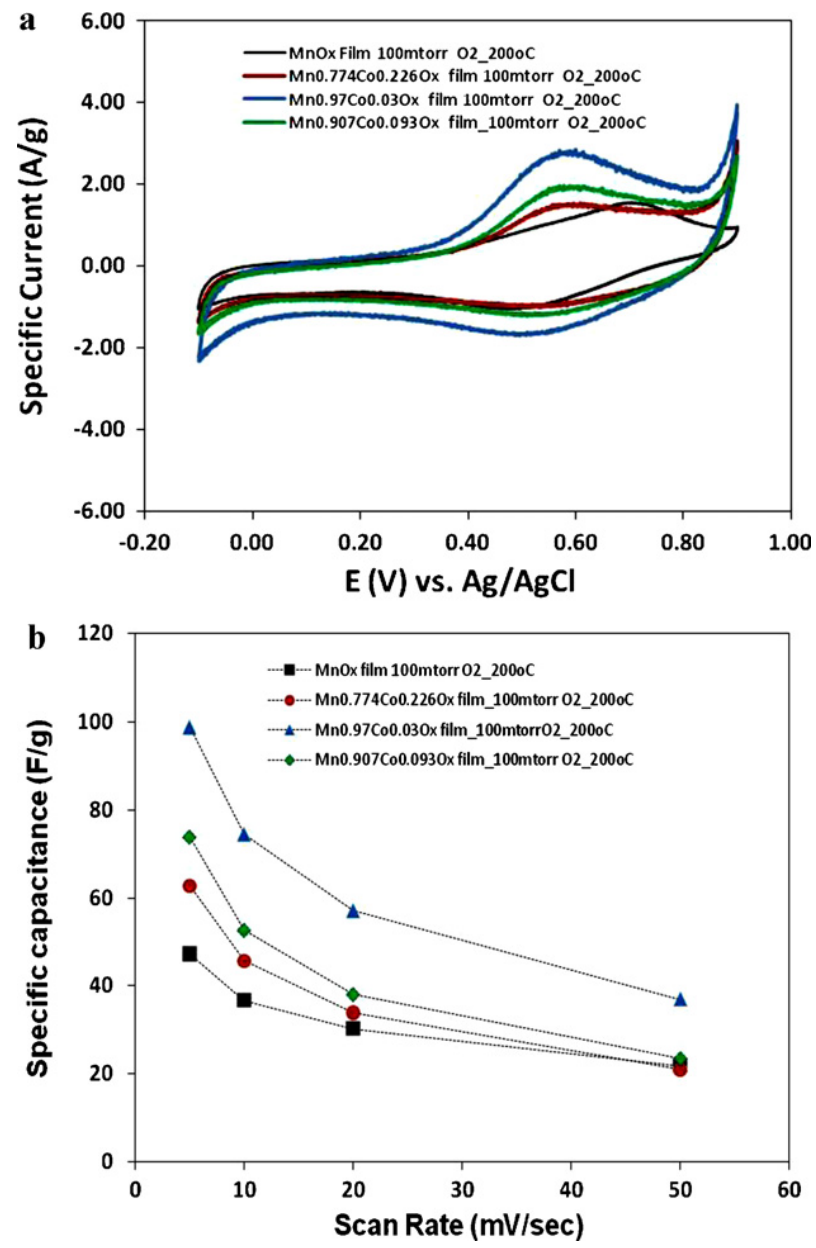

Figure 2. Cyclic voltammetry (a) and specific capacitance (b) of amorphous $\mathrm{MnO}_{x}$ film and various Co-doped amorphous $\mathrm{MnO}_{x}$ films deposited by PLD at 200॰C in 100 mTorr of $\mathrm{O}_{2}$ (from ref. 6).

Spinel nickel cobaltite (doped or un-doped, such as $\mathrm{NiCo}_{2} \mathrm{O}_{4}$ and $\mathrm{NiMn}_{\mathrm{x}} \mathrm{Co}_{2-\mathrm{x}} \mathrm{O}_{4-\mathrm{y}}(\mathrm{x} \leq 1.0)$ ) possesses much better electronic conductivity than that of $\mathrm{NiO}$ and $\mathrm{Co}_{3} \mathrm{O}_{4}$. They are low-cost and have multiple oxidation states, and therefore, are also exploited for supercapacitor ap- 
plications. C. Wang et al. [10] prepared nanostructured $\mathrm{NiCo}_{2} \mathrm{O}_{4}$ spinel platelet like particles with narrow size distribution of $5-10 \mathrm{~nm}$ by co-precipitation process. The $\mathrm{NiCo}_{2} \mathrm{O}_{4}$ has excellent conductivity and showed a high-specific capacitance of $671 \mathrm{~F} \mathrm{~g}^{-1}$ under a mass loading of $0.6 \mathrm{mg} \mathrm{cm}^{-1}$ at a current density of $1 \mathrm{~A} \mathrm{~g}^{-1}$. Chang et al. [11] also prepared $\mathrm{NiCo}_{2} \mathrm{O}_{4}$ and $\mathrm{NiMn}_{\mathrm{x}} \mathrm{Co}_{2-\mathrm{x}} \mathrm{O}_{4-\mathrm{y}}(\mathrm{x} \leq 1.0)$ using a precipitation route. They found that the spinel structural of $\mathrm{NiCO}_{2} \mathrm{O}_{4}$ is retained with a quarter of the Co ions replaced with $\mathrm{Mn}$. The presence of $\mathrm{Mn}$ significantly suppresses crystallite growth upon thermal treatment, and greatly enhances the specific capacitance of the spinel. At the scan rate of $4 \mathrm{mV} \mathrm{s}^{-1}$, the specific capacitance is found to increase from $30 \mathrm{~F} \mathrm{~g}^{-1}$ for Mn content $\mathrm{x}=0$ to $110 \mathrm{~F} \mathrm{~g}^{-1}$ for $\mathrm{x}=0.5$. The $\mathrm{NiMn}_{0.5} \mathrm{Co}_{1.5} \mathrm{O}_{4}$ powder has been found by the authors to be much smaller surface area than the $\mathrm{NiCo}_{2} \mathrm{O}_{4}$ powder. Therefore, the remarkable capacitance enhancement exhibited by the $\mathrm{NiMn}_{0.5} \mathrm{Co}_{1.5} \mathrm{O}_{4}$ electrode is not due to microstructural variations of the oxide powders. The capacitance enhancement is attributed by the authors to the facile charge-transfer characteristic of the Mn ions, which enables a greater amount of charge transferred between the oxide and the aqueous electrolyte species over the same potential window.

\subsection{Carbon nanotubes based nanocomposites}

Carbon nanotubes (CNTs) have superior material properties such as high chemical stability, aspect ratio, mechanical strength and activated surface area as well as outstanding electrical properties, which make them good electroactive material candidates for supercapacitors. The electrodes made from CNTs exhibit a unique pore structure for change storage; however, there are limitations for further increasing the effective surface area of the CNTs, as well as relatively high materials cost which limit the commercial application of CNTs based supercapacitors. To improve the performance of CNTs, they are composited with conductive polymers and metal oxides. This section will summarize the recent development of CNTs based nanocomposites for supercapacitor applications.

\subsubsection{Carbon nanotubes and polymer nanocomposites}

Techniques that can be used to synthesize CNTs include Arc discharge, chemical vapour deposition, and laser ablation. Kay et al. [12] synthesized single-walled CNTs by dc arc discharge of a graphite rod under helium gas using $\mathrm{Ni}, \mathrm{Co}$, and $\mathrm{FeS}$ as catalysts. Then they prepared single-walled CNTs-polypyrrole (PPY) nanocomposite using in situ chemical polymerization of pyrrole monomer in solution with single-walled CNTs suspension. Figure 3 shows the FE-SEM images of as-grown single-walled CNTs, pure PPY, and single-walled CNT-PPY nanocomposite powder formed by the in situ chemical polymerization. The asgrown single-walled CNTs are randomly entangled and cross-linked, and some carbon nanoparticles are also observed, as shown in Figure 3(a). In Figure 3(b), the image for pure PPY synthesized without single-walled CNTs present in solution shows a typical granular morphology with granule size of about $0.2-0.3 \mathrm{~mm}$. Figure 3(c) demonstrates that the individual carbon nanotube bundles are uniformly coated with PPY which indicates that in situ chemical polymerization of pyrrole can effectively coated all the CNTs. The electrode prepared using single-walled CNTs-PPY nanocomposite as active materials show very high 
specific capacitance: a maximum specific capacitance of $265 \mathrm{~F} \mathrm{~g}^{-1}$ from the single-walled CNT-PPY nanocomposite electrode containing $15 \mathrm{wt}$. \% of the conducting agent was obtained. Figure 4 shows the specific capacitances of the as-grown single-walled CNTs, pure PPY, and single-walled CNTs-PPY nanocomposite electrodes as a function of discharge current density. In comparison to the pure PPY and as-grown single-walled CNTs electrodes, the single-walled CNTs-PPY nanocomposite electrode shows very high specific capacitance. The improvement in the specific capacitance of the CNTs-PPY nanocomposite was attributed by the authors to the increase in active surface area of pseudocapacitive PPY by CNTs. CNTs was also composited with polyaniline (PANI) by Deng et al. [13]. In their experiments, the CNTs-PANI was prepared using direct polymerization of aniline monomer with oxidant agent, $\left(\mathrm{NH}_{4}\right)_{2} \mathrm{~S}_{2} \mathrm{O}_{8}$, in acidic solution containing CNTs suspension, similar to the CNTs-PPY nanocomposite prepared by Kay et al. The CNTs-PANI nanocomposite achieved a specific capacitance of $183 \mathrm{~F} \mathrm{~g}^{-1}$, almost 4 times higher than pure CNTs $\left(47 \mathrm{~F} \mathrm{~g}^{-1}\right)$.
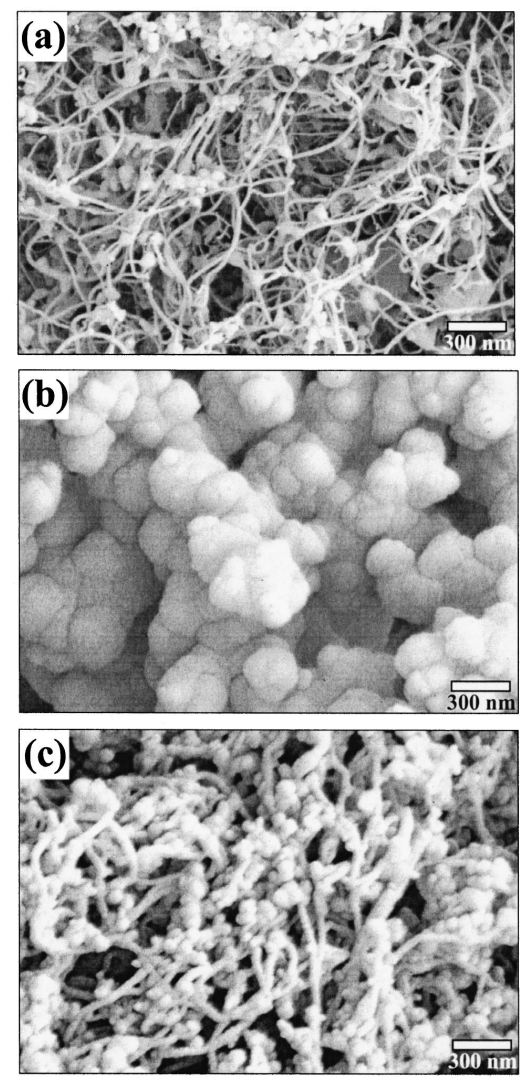

Figure 3. The FE-SEM images of (a) as-grown single-walled CNTs, (b) pure PPY, and (c) single-walled CNTs-PPY powder (from ref. 12) 


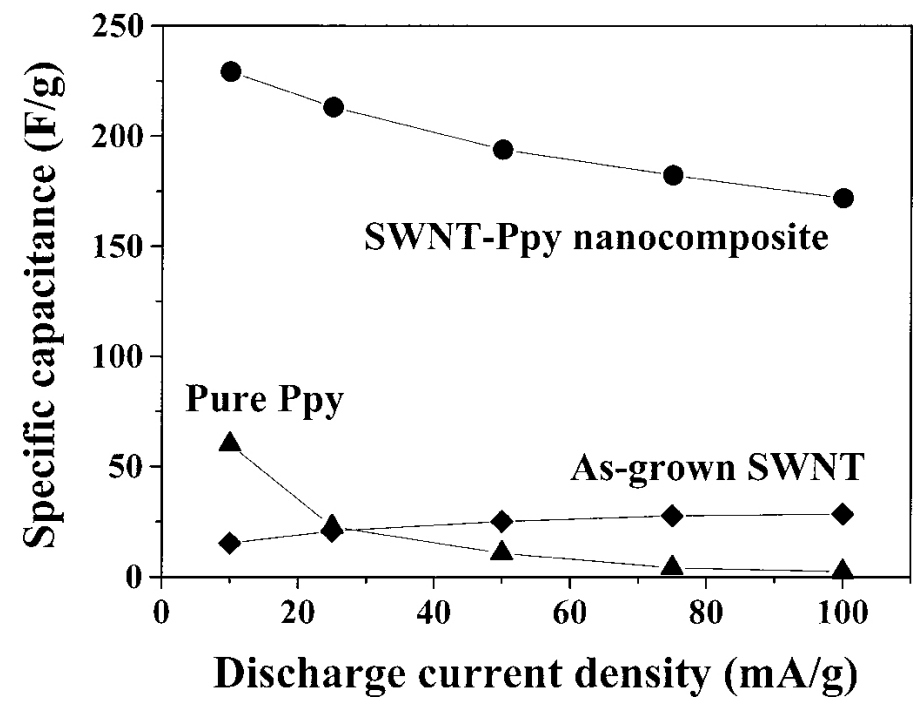

Figure 4. The specific capacitances of the as-grown single-walled CNTs, pure PPY, and single-walled CNTs-PPY, singlewalled CNTs-PPY nanocomposite electrodes as a function of ischarge current density at a charging voltage of $0.9 \mathrm{~V}$ for $10 \mathrm{~min}$ (from ref.

\subsubsection{Carbon nanotubes and ruthenium oxide nanocomposites}

Although $\mathrm{RuO}_{2}$ shows remarkable performance as supercapacitors electrode active materials, its high cost has limited its commercial applications. To fully utilize the expensive $\mathrm{RuO}_{2}$ in an electrode, it is necessary to disperse it over high surface area materials such as CNTs. Y-T. Kim et al. $[14,15]$ discovered a new way to uniformly disperse $\mathrm{RuO}_{2}$ over the whole surface area of CNTs: firstly, they oxidized the multi-walled CNTs in a concentrated $\mathrm{H}_{2} \mathrm{SO}_{4}-$ $\mathrm{HNO}_{3}$ mixture to introduce the surface carboxyl groups, and then they prepared multi-walled CNTs- $\mathrm{RuO}_{2}$ nanocomposites with a conventional sol-gel method. The surface carboxyl groups formed on CNTs allow $\mathrm{RuO}_{2}$ to disperse more effectively since bond formation between $\mathrm{RuO}_{2}$ and carboxyl group protects against agglomeration of $\mathrm{RuO}_{2}$ as illustrated in Figure 5. Figure 5 schematically shows that for purified multi-walled $\mathrm{CNTs}, \mathrm{RuO}_{2}$ can be spontaneously reduced to metallic Ru on the CNTs surface and subsequently covered with $\mathrm{RuO}_{\mathrm{x}}(\mathrm{OH})_{\mathrm{y}}$ via the reaction with $\mathrm{NaOH}$ to form core-shell structures. For oxidized CNTs, the positive charged Ru precursor ions have limited contact with the CNTs surface to be reduced due to negatively charged carboxyl groups. Surface carboxyl groups act not only as protectors against spontaneous reduction of $\mathrm{Ru}$ ions but also as anchorage centres for $\mathrm{Ru}$ which enhance the dispersity of $\mathrm{RuO}_{2}$ and hinder their agglomeration into large particles. TEM images in figure 5 show the dramatic difference in particle size of $\mathrm{RuO}_{2}$ nanoparticle and dispersity between $\mathrm{RuO}_{2}$-pure CNTs and $\mathrm{RuO}_{2}$-oxidized CNTs. 


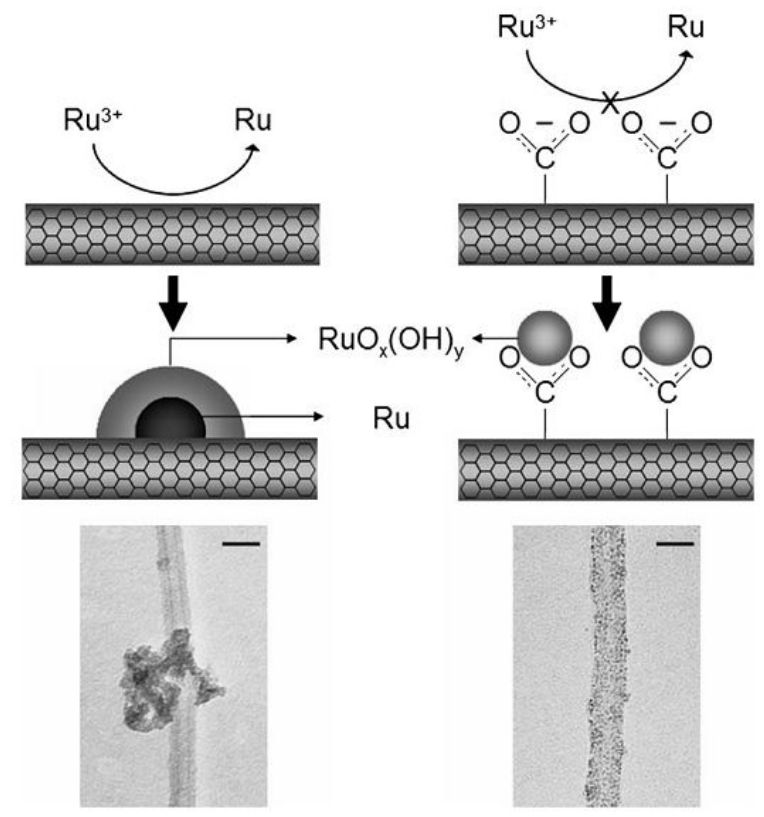

Figure 5. Schematic diagram of the different formation mechanisms of $\mathrm{RuO}_{2}$ on purified multi-walled CNTs and oxidized multi-walled CNTs in the preparation process and their actual TEM images scale bar (20 nm). (from ref. 15)

Another way to effectively disperse $\mathrm{RuO}_{2}$ over CNTs was developed by Hsieh et al. [16] who grew vertically aligned multi-walled CNTs films directly by CVD on the Ti current collector using thin nickel layers as the catalyst. Hydrous ruthenium dioxide was then directly deposited onto the surface of CNTs electrodes by electrochemical CV deposition from an aqueous acidic solution of ruthenium trichloride $\left(\mathrm{RuCl}_{3} \cdot \mathrm{nH}_{2} \mathrm{O}\right)$. The SEM morphology of the composites shows that the surfaces of the multi-walled CNT/Ti electrodes were coated uniformly with hydrous ruthenium dioxide, which increased the utilization of the electroactive $\mathrm{RuO}_{2}$ material. Electrochemical measurements showed that the $\mathrm{RuO}_{2} \cdot \mathrm{nH}_{2} \mathrm{O}-\mathrm{CNT}$ s nanocomposites have high capacitance of $1652 \mathrm{~F} \mathrm{~g}^{-1}$ and decay rate of $3.45 \%$ at $10 \mathrm{mV} \mathrm{s}^{-1}$ in a $1.0 \mathrm{M}$ $\mathrm{H}_{2} \mathrm{SO}_{4}$ aqueous electrolyte within the potential range from -0.1 to $1.0 \mathrm{~V}$. Figure 6 shows the comparison of specific capacitance of $\mathrm{RuO}_{2} \cdot \mathrm{nH}_{2} \mathrm{O}$-CNTs nanocomposite electrode with $\mathrm{RuO}_{2} \cdot \mathrm{nH}_{2} \mathrm{O}$ and multi-walled CNTs electrodes at a scan rate of $10 \mathrm{mV} / \mathrm{s}$. The results in the figure 6 clearly show that the capacitance of $\mathrm{RuO}_{2} \cdot \mathrm{nH}_{2} \mathrm{O}-\mathrm{CNTs}$ was much higher (4.7 times) than those of pure materials. Chemical impregnation of ruthenium nitrosylnitrate solution $\left(\mathrm{Ru}(\mathrm{NO})\left(\mathrm{NO}_{3}\right)_{\mathrm{x}}(\mathrm{OH})_{\mathrm{y}}\right.$ on the CVD-grown multi-walled CNTs following by calcination at $350 \circ \mathrm{C}$ process was used by Lee et al. [17] to form $\mathrm{RuO}_{2}-\mathrm{CNT}$ nanocomposites. The specific capacitance of the nanocomposite was found to be as high as $628 \mathrm{~F} \mathrm{~g}^{-1}$. The authors believed that nanoporous three-dimensional structure of $\mathrm{RuO}_{2}-\mathrm{CNTs}$ nanocomposite facilitated the electron and ion transfer. Byung Chul Kim et al. [18] used the electrochemical potentiody- 
namic deposition method to prepare $\mathrm{RuO}_{2}-\mathrm{CNTS}$ and $\mathrm{Ru} / \mathrm{Co}$ oxides-CNTs nanocomposites from $\mathrm{RuCl}_{3}$, and $0.1 \mathrm{M} \mathrm{CoCl} 2+0.05 \mathrm{M} \mathrm{RuCl}_{3}$ solutions, respectively. All the composites showed considerable increase in capacitance values. The Ru/Co mixed oxides-CNTs showed superior performance $\left(570 \mathrm{~F} \mathrm{~g}^{-1}\right)$ at high scan rates $\left(500 \mathrm{mV} \mathrm{s}^{-1}\right)$ when compared to the $\mathrm{RuO}_{2}$ electrode $\left(475 \mathrm{~F} \mathrm{~g}^{-1}\right)$. This increase in capacitance at high scan rates is attributed by the authors to the enhanced electronic conduction of Co in the composites.

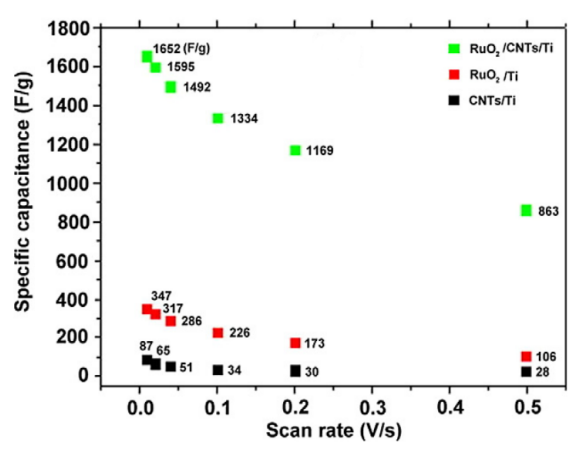

Figure 6. Specific capacitance of $\mathrm{RuO}_{2} \cdot \mathrm{nH}_{2} \mathrm{O}-\mathrm{CNT}$, $\mathrm{RuO}_{2} \cdot \mathrm{nH}_{2} \mathrm{O}$, and multi-walled CNTs after 80 scan cycles with scan rates from 10 to $500 \mathrm{mV} / \mathrm{s}$ (from ref. 16).

\subsubsection{Carbon nanotubes and other metal oxide nanocomposites}

Besides $\mathrm{RuO}_{2}$, there are many other metal oxides that were composited with CNTs to form electroactive materials for supercapacitors. Chen et al. [19] used thermal CVD to grow multi-walled CNTs on a stainless steel plate and then on top of $\mathrm{CNTs}, \mathrm{IrO}_{2}$ nanotubes were deposited using metal-organic CVD with the iridium source of $\left(\mathrm{C}_{6} \mathrm{H}_{7}\right)\left(\mathrm{C}_{8} \mathrm{H}_{12}\right) \mathrm{Ir}$ at $350 \circ \mathrm{C}$. The $\mathrm{IrO}_{2}$ square nanotube crystals were grown on the upper section of the CNTs thin film. Figure 7 shows the morphologies of multi-walled $\mathrm{CNTs}, \mathrm{IrO}_{2}$ nanotubes, and $\mathrm{IrO}_{2}$ nanotubes multi-walled CNTs nanocomposite. The cross-sectional view of multi-walled CNTs, figure 7(b), shows that there are upper and lower sections in the CNTs thin film. The upper section, $\sim 2 \mu \mathrm{m}$ in thickness, consists of entangled carbon nanotubes without distinct orientation. The lower section, approximately $4 \mu \mathrm{m}$ thick, is composed of largely parallel nanotubes, aligned in the vertical direction. These nanotubes act as the templates for the $\mathrm{IrO}_{2}$ nanotube growth. Figures 7(c) and (d) show a top view and cross-sectional view of $\mathrm{IrO}_{2}$ nanotubes grown on stainless steel substrate. Figure 7(e) and (f) show $\mathrm{IrO}_{2}$ nanotubes grown over CNTs. Figure 7(e) indicates that the grown $\mathrm{IrO}_{2}$ nanotubes had a high density along the wires of multi-walled CNTs in the upper section. In comparison to multi-walled CNTs, the nanostructured $\mathrm{IrO}_{2}-\mathrm{CNTs}$ increases the capacitance by a factor of six, from 15 to $69 \mathrm{~F} \mathrm{~g}^{-1}$, and reduces the resistance from 90 to $60 \Omega$. Such a hierarchical structure provides a high surface area for electrical charge storage, and a double-layer capacitance in conjunction with 
pseudocapacitance. Electrochemical anodic deposition of $\mathrm{MnO}_{\mathbf{x}} \bullet \mathrm{nH}_{2} \mathrm{O}$ films from $\mathrm{MnSO}_{4} \bullet 5 \mathrm{H}_{2} \mathrm{O}$ solution on CNTs coated Ni substrates was used by Lee et al. [20] to form amorphous $\mathrm{MnO}_{\mathrm{x}}-\mathrm{CNTs}$ nanocomposite electrode. The CNTs were electrophoretically deposited on the Ni substrate by applying a dc voltage of $20 \mathrm{~V}$ before the deposition of $\mathrm{MnOx} \bullet \mathrm{nH} 2 \mathrm{O}$. The $\mathrm{MnO}_{\mathrm{x}}-\mathrm{CNT}$ nanocomposite electrodes have shown much better energy storage capabilities than $\mathrm{MnO}_{\mathrm{x}}$ deposited directly on the Ni substrate: the specific capacitances were 415 as obtained from CV measurements with a scan rate of $5 \mathrm{mV} / \mathrm{s}$ and it preserved $79 \%$ of its original capacitance value after 1000 cycles. The authors attributed the improvement to the low resistance and large surface area of the nanocomposite electrodes. Other metal oxide CNTs nanocomposites being investigated include NiO-CNTs[21], $\mathrm{V}_{2} \mathrm{O}_{5}-\mathrm{CNTs}$ [22] and $\mathrm{SnO}_{2}-\mathrm{V}_{2} \mathrm{O}_{5}-\mathrm{CNTs}$ [23]. The $\mathrm{V}_{2} \mathrm{O}_{5}-\mathrm{CNTs}$ composite was prepared by electrochemical deposition of $\mathrm{V}_{2} \mathrm{O}_{5}$ on vertically aligned multi-walled CNTs and it can reach a specific capacitance of $713.3 \mathrm{~F} \mathrm{~g} \mathrm{~g}^{-1}$ at $10 \mathrm{mV} \mathrm{s}^{-1}$. The $\mathrm{SnO}_{2}-\mathrm{V}_{2} \mathrm{O}_{5}-\mathrm{CNTs}$ was prepared by simply mixing CNTs and $\mathrm{SnO}_{2}-\mathrm{V}_{2} \mathrm{O}_{5}$ mixed oxide powder in a mortar prior and fixing on the surface of a graphite electrode that was impregnated with paraffin. At a scan rate of $100 \mathrm{mV} \mathrm{s}^{-1}$, the $\mathrm{SnO}_{2}-\mathrm{V}_{2} \mathrm{O}_{5}-\mathrm{CNT}$ s electrode provides $121.4 \mathrm{~F} \mathrm{~g}^{-1}$ specific capacitance.

Other types of high surface area carbon materials such as activated carbons, carbon fibres and carbon aerogels were also composited with metal oxide to form high performance electroactive materials. Those examples include vapour-grown carbon fibre (VGCF) - $\mathrm{RuO}_{2}$ $\mathrm{xH}_{2} \mathrm{O}$ nanocomposite prepared by a thermal decomposition [24], $\mathrm{RuO}_{2} \cdot \mathrm{xH}_{2} \mathrm{O}$-mesoporous carbon nanocomposites prepared using impregnation [25], $\mathrm{ZnO}$-activated carbon nanocomposite electrode by simply mixing [26] and $\mathrm{MoO}_{3}$-graphite prepared by ball milling [27].

\subsection{Pseudocapacitive polymer and metal oxide nanocomposites}

Electronically conducting polymers derived from monomers such as pyrrole, aniline, and thiophene have unique properties, such as good environmental stability, electroactivity, and unusual doping/de-doping chemistry, therefore, they are suitable for active electrode material usage in supercapacitors. When used as electrode materials, these polymers have advantage over carbon-based materials since they have both electrochemical double layer capacitance and pseudocapacitance which arises mainly from the fast and reversible oxidation and reduction processes related to the $\pi$-conjugated polymer chain. However, conducting polymers have problems of typical volumetric shrinkage during ejection of ions (doped ions) and low conductance at de-doped state which would result in high ohmic polarization of supercapacitors. In order to solve this problem, conducting polymers were mixed with metal oxides to form nanocomposites and the synergistic effect of the polymer-metal oxide nanocomposites has been exploited. Such nanocomposites were found to have the advantages of polymers such as flexibility, toughness and coatability and metal oxides such as hardness, and durability. They also possess some synergetic properties which are different from that of parent materials. This section will summarize the recent development in this serial of materials. 


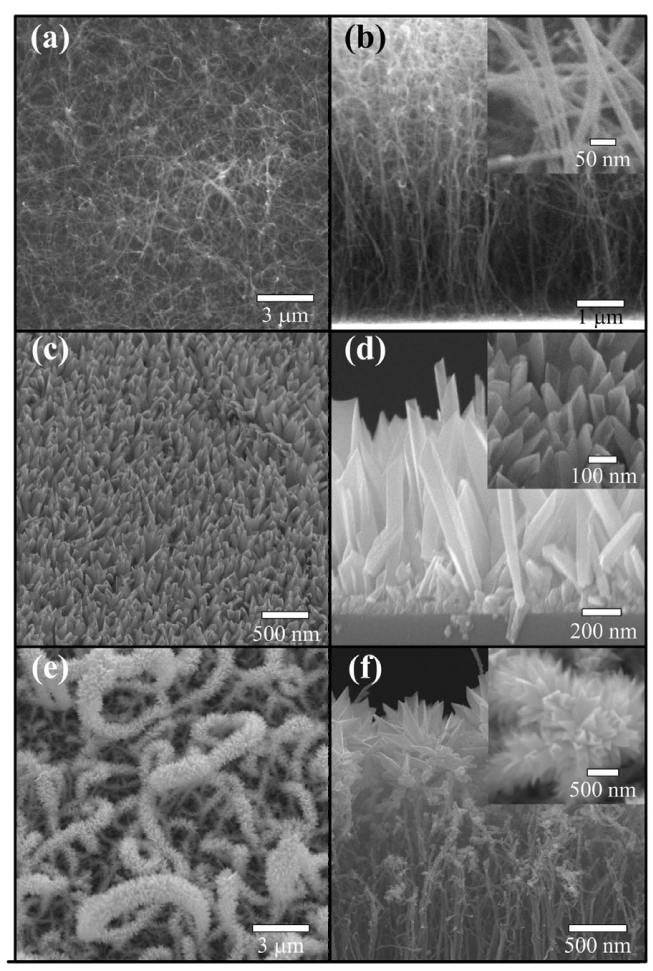

Figure 7. SEM image of CNTs top view (a), CNTs cross-sectional view (b), IrO 2 nanotubes top view (c), IrO 2 cross-sectional view (d), $\mathrm{IrO}_{2}-\mathrm{CNTS}$ top view (e), $\mathrm{IrO}_{2}-\mathrm{CNTS}$ cross-sectional view (f). The inset of (b) and (d) are magnified images. (from ref. 19).

\subsubsection{Polyaniline (PANI) and metal oxide nanocomposites}

Polyaniline (PANI) is one of the most important conducting polymers because of its ease of synthesis at low cost, good processability, environmental stability and easily tuneable conducting properties. The synthesis and studies of composites of PANI and metal oxides such as $\mathrm{MnO}_{2}, \mathrm{SnO}_{2}$ and $\mathrm{MnWO}_{4}$ have been carried out. In a PANI-metal oxide nanocomposite, PANI not only serves as an electroactive material for energy storage but also as a good coating layer to restrain metal oxides from dissolution in acidic electrolytes. Chen et al. [28] synthesized a very high performance $\mathrm{PANI}-\mathrm{MnO}_{2}$ nanocomposite using the following procedure: first, the hydroxylated $\mathrm{MnO}_{2}$ nanoparticles were surface modified with silane coupling agent, $\mathrm{ND} 42$, then the obtained surface modified $\mathrm{MnO}_{2}$ nanoparticles (ND- $\mathrm{MnO}_{2}$ ) were washed and dried. Electro-co-polymerization of aniline and $\mathrm{ND}-\mathrm{MnO}_{2}$ nanoparticles was conducted on a carbon cloth in an electrolyte solution containing $\mathrm{ND}-\mathrm{MnO}_{2}$, aniline, $\mathrm{H}_{2} \mathrm{SO}_{4}$ and $\mathrm{Na}_{3} \mathrm{PO}_{3}$. The co-polymerization was preceded through successive cyclic voltammetric scans. The whole synthesis process is illustrated in Figure 8. Electro-co-polymerization method was also used to prepare unmodified $\mathrm{PANI}-\mathrm{MnO}_{2}$ nanocomposite and pure 
PANI. The SEM images of PANI-MnO ${ }_{2}$ PANI-ND-MnO ${ }_{2}$ films reveal that the addition of $\mathrm{MnO}_{2}$ nanoparticles promotes the one dimensional growth of PANI, which substantially reduces the size of the nanorods and increases the surface area/internal space of the composite films (Figure 9). PANI-ND- $\mathrm{MnO}_{2}$ composite film has an average specific capacitance of $\sim 80$ $\mathrm{F} \mathrm{g}^{-1}$ and a very stable coulombic efficiency of $\sim 98 \%$ over 1000 cycles. It also exhibit high intrinsic electrical conductivity and good kinetic reversibility. The excellent properties were attributed by the authors to the improved interaction between $\mathrm{MnO}_{2}$ and PANI and the increased effective surface area in PANI-ND- $\mathrm{MnO}_{2}$ film, due to the surface modification of $\mathrm{MnO}_{2}$ nanoparticles with the silane coupling reagent. Significantly high specific capacitor was achieved with PANI-SnO $\mathrm{S}_{2}$ nanocomposites prepared by Hue et al. [29] using a chemical method in which $\mathrm{SnO}_{2}$ nanoparticles and aniline were dispersed in sodium dodecylbenzenesulfonate solution and then, ammonium persulfate was added to the above mixture to start polymerization. The PANI-SnO $\mathrm{S}_{2}$ nanocomposite thus prepared had a high specific capacitance of $305.3 \mathrm{~F} \mathrm{~g}^{-1}$ with a specific energy density of $42.4 \mathrm{Wh} \mathrm{kg}^{-1}$ and a coulombic efficiency of $96 \%$. The energy storage density of the composite was about three times as compared with pure $\mathrm{SnO}_{2}$.

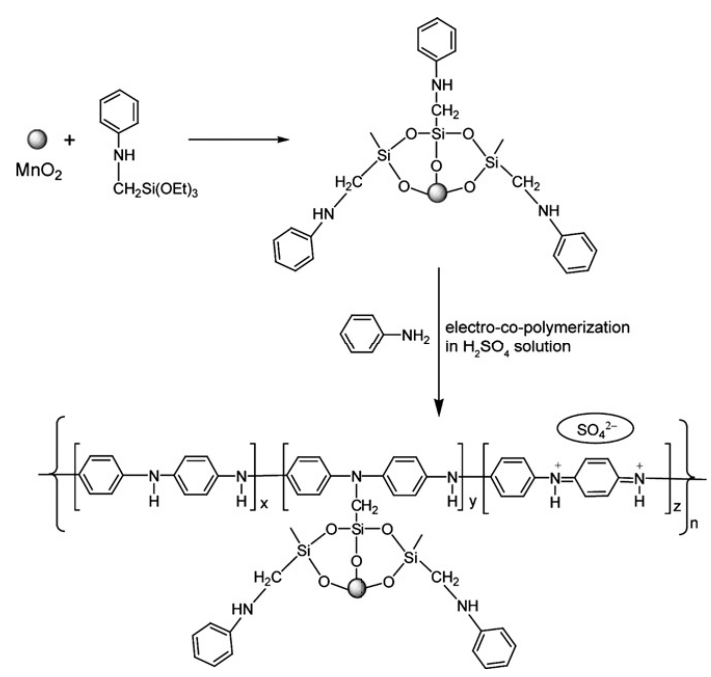

Figure 8. A schematic diagram illustrates the reaction pathway for the synthesis of PANI-ND-MnO ${ }_{2}$ nanocomposite film (from ref. 28).

Wang et al. [30] developed an innovative way to synthesize PANI- $\mathrm{MnO}_{2}$ nanocomposites. This so-called "interfacial synthesis" utilized the interfacial region between an organic phase and an aqueous phase to synthesize the composite. The organic phase was prepared by dissolving aniline monomers into inorganic Trichloromethane $\left(\mathrm{CHCl}_{3}\right)$ solution, while the aqueous phase was obtained by dissolving potassium permanganate in distilled water. When the aqueous solution was added into the organic solution, an interface was formed immediately between the two phases and the reaction occurred. During the reaction, aniline 
was diffused from the organic solution to the interface and was chemically oxidized into polyaniline. At the same time, $\mathrm{MnO}_{4}{ }^{-}$was reduced to manganese oxide precipitate. Finally, the PANI- $\mathrm{MnO}_{2}$ nanocomposite was formed and remained in the aqueous solution. For comparison, conventional chemical co-precipitation of $\mathrm{MnO}_{2}-\mathrm{PANI}$ composite was performed to make the conventional PANI- $\mathrm{MnO}_{2}$ composite. Both synthesis processes was schematically illustrated in Figure 10. The interfacial synthesized $\mathrm{MnO}_{2}-\mathrm{PANI}$ composite shows larger specific surface area $\left(124 \mathrm{~m}^{2} \mathrm{~g}^{-1}\right)$ and more uniform pore-size distribution than the composite prepared by chemical co-precipitation as shown in Figure 11. It exhibits a higher specific capacitance of $262 \mathrm{~F} \mathrm{~g}^{-1}$ (about twice amount of conventionally prepared $\mathrm{MnO}_{2}$-PANI composite) with better cycling stability. The authors attributed the observed enhanced electrochemical properties of the interfacial synthesized $\mathrm{MnO}_{2}$-PANI composite electrode to its unique hollow microstructure with well-defined mesoporosity and the coexistence of conducting PANI. Other interesting PANI metal oxide nanocomposites include

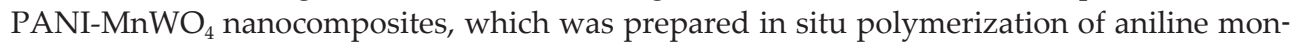
omer in solution containing $\mathrm{MnWO}_{4}$ nanoparticles [31]. The composite has shown good electrochemical properties: with $50 \%$ of $\mathrm{MnWO}_{4}$ loading, the $\mathrm{PANI}-\mathrm{MnWO}_{4}$ nanocomposites shows high specific capacitance of $475 \mathrm{~F} \mathrm{~g}^{-1}$, much higher than that of the physical mixture of PANI and $\mathrm{MnWO}_{4}\left(346 \mathrm{~F} \mathrm{~g}^{-1}\right)$.

\subsubsection{Other polymer and metal oxide nanocomposites}

Beside Polyaniline (PANI), other conducting polymers that have been used to composite with metal oxides to form electroactive materials including polypyrrole (PPY), polythiophene and their derivatives such as Poly 3,4-ethylenedioxythiophene (PEDOT). PEDOT is a stable and environmentally friendly polymer and has controllable electrical conductivity. However, PEDOT suffers from problems such as volumetric swelling and shrinkage during the insertion and ejection of ions. PEDOT was comprised with pseudocapacitive metal oxides such as $\mathrm{MnFe}_{2} \mathrm{O}_{4}, \mathrm{CoFe}_{2} \mathrm{O}_{4}$ and $\mathrm{MoO}_{3}$ to improve its property. The synergistic effect of composite formation plays a significant role to increase the capacitance value. Sen et al. [32] prepared PEDOT- $\mathrm{NiFe}_{2} \mathrm{O}_{4}$ nanocomposites by chemical polymerization of EDOT monomer in solution containing nickel ferrite nanoparticles $\left(\mathrm{NiFe}_{2} \mathrm{O}_{4}\right)$. Pure PEDOT polymer in both n-hexane medium and aqueous medium was also synthesized by similar procedure in the absence of $\mathrm{NiFe}_{2} \mathrm{O}_{4}$ nanoparticles. Electrochemical CVs and impedance spectroscopy were used to characterize the PEDOT- $\mathrm{NiFe}_{2} \mathrm{O}_{4}$ nanocomposite as well as pure PEDOT synthesized in organic medium and aqueous medium, and $\mathrm{NiFe}_{2} \mathrm{O}_{4}$ nanoparticles prepared by sol-gel procedure. Figure 12 shows typical Nyquist impedance spectra of the four compounds over a frequency range of $10 \mathrm{kHz}-10 \mathrm{mHz}$ with a potential amplitude of $5 \mathrm{mV}$. The impedance results show that introduction of $\mathrm{NiFe}_{2} \mathrm{O}_{4}$ nanoparticles into the PEDOT not only helps to reduce the intrinsic resistance (the intercept of the semi-circle with real axis $\left(Z^{\prime}\right)$ at high frequencies is the measure of internal resistance) through the development more mesoporous structures but also increase the kinetics of electron transfer through redox process leading to the enhancement of pseudocapacitance in the composite materials (pseudocapacitance values were also determined from the impedance by fittings the spectra with Randles equivalent circuit). The PEDOT- $\mathrm{NiFe}_{2} \mathrm{O}_{4}$ nanocomposite shows high specific capacitance $\left(251 \mathrm{~F} \mathrm{~g}^{-1}\right)$ in comparison to $\mathrm{NiFe}_{2} \mathrm{O}_{4}\left(127 \mathrm{~F} \mathrm{~g}^{-1}\right)$ 
and PEDOT (156 F g-1) where morphology of the pore structure was believed to play a significant role over the total surface area. PEDOT was also composited with $\mathrm{MoO}_{3}$ by Murugan et al. [33] using chemical polymerization of EDOT monomer with $\mathrm{FeCl}_{3}$ as oxidizing agent in $\mathrm{MoO}_{3}$ suspension. The nanocomposite also has much higher specific capacitance $\left(300 \mathrm{~F} \mathrm{~g}^{-1}\right)$ compared to that of pristine $\mathrm{MoO}_{3}\left(40 \mathrm{mF} \mathrm{g}^{-1}\right)$. The improved electrochemical performance was attributed by the authors to the intercalation of electronically conducting PEDOT between $\mathrm{MoO}_{3}$ layers and an increase in surface area.

(a)

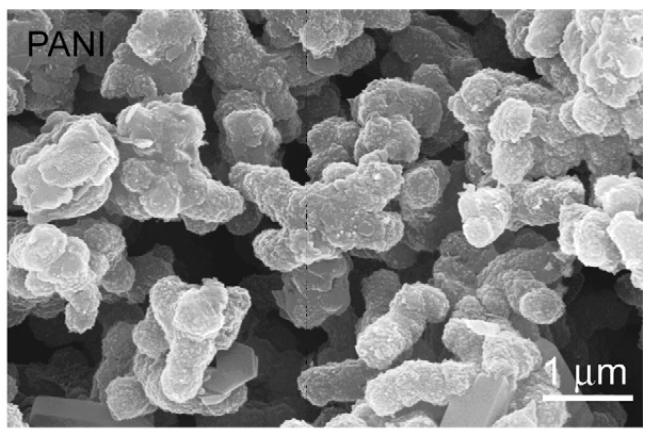

(b)

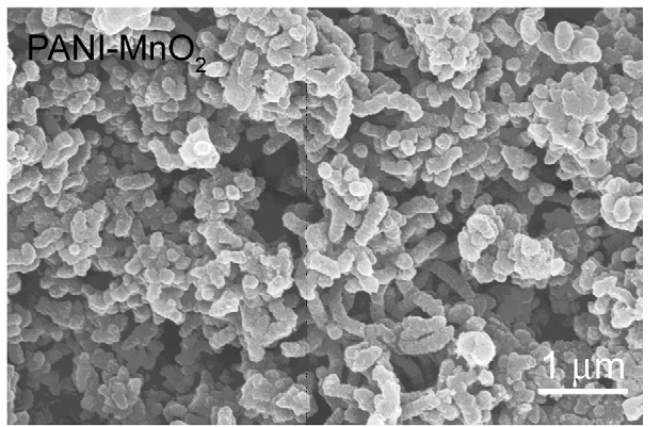

(c)

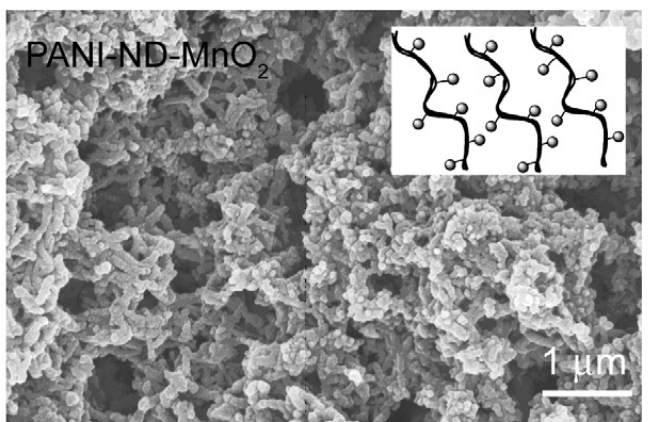

Figure 9. SEM images of (a) PANI, (b)PANI-MnO ${ }_{2}$ and (c)PANI-ND-MnO ${ }_{2}$ composite(from ref. 28). 
a

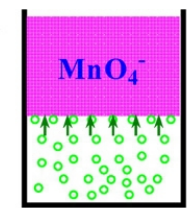

$\circ$ aniline monomer

$\mathrm{b}$

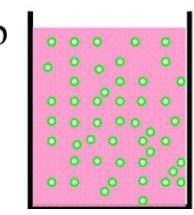

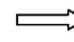
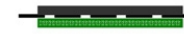

$\mathrm{MnO}_{2}$

- polyaniline

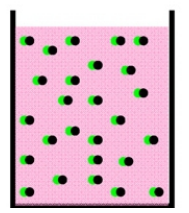

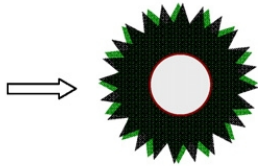

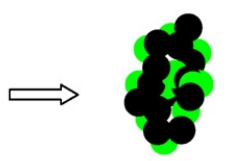

Figure 10. Schematic illustration of the formation mechanisms of $\mathrm{MnO}_{2}$-PANI composites: (a) interfacial synthesis and (b) chemical co-precipitation (from ref. 30).

Polypyrrole (PPY) is also a promising conducting polymer material due to its highly reversible redox reaction. Although the electrical conductivity of intrinsic PPY is low, doping of surfactants can enhance effectively the electrical conductivity of PPY. p-Toluenesulfonic acid (p-TSA) was used as a dopant by Dong et al. [34] to prepare $\mathrm{MnO}_{2}-\mathrm{PPY} / \mathrm{TSA}$ nanocomposite for supercapacitor applications. TSA and pyrrole were dispersed ultrasonically in deionised water to form a homogeneous solution. With the addition of $\mathrm{KMnO}_{4}$ or $\mathrm{FeCl} 3 \bullet 6 \mathrm{H} 2 \mathrm{O}$ oxidant, redox reactions occurred and $\mathrm{MnO}_{2}$-PPY/TSA nanocomposite was produced. Micrographs and BET isotherm measurements showed that the particle and the pore size of the $\mathrm{MnO}_{2}$-PPY/TSA nanocomposite are much smaller than those of the $\mathrm{MnO}_{2}-\mathrm{PPY}$. Electrochemical measurements showed that the $\mathrm{MnO}_{2}-\mathrm{PPY} / \mathrm{TSA}$ nanocomposite electrode exhibited a higher specific capacitance of $\sim 376 \mathrm{~F} \mathrm{~g}^{-1}$ at $3 \mathrm{~mA} \mathrm{~cm}^{-2}$ and better cycling stability in $0.5 \mathrm{M} \mathrm{Na}_{2} \mathrm{SO}_{4}$ solution than the $\mathrm{MnO}_{2}$-PPY. Another polymer metal oxide composite that shows promising supercapactive properties is $\mathrm{MnO}_{2}$-poly(aniline-co-o-anisidine) [35], which has specific capacitance of the $262 \mathrm{~F} \mathrm{~g}^{-1}$ in $1 \mathrm{M} \mathrm{Na}_{2} \mathrm{SO}_{4}$ at a current density of $1 \mathrm{~A} \mathrm{~g}^{-1}$. All the above results presented by various authors have demonstrated that the development of novel metal oxide-conducting polymers nanocomposite holds great potential applications in high-performance electrochemical capacitors.

\subsection{Graphene based nanocomposites}

Graphene (GN) is a two-dimensional monolayer of $\mathrm{sp}^{2}$-bonded carbon atoms. It has attracted increasing attention in recent years, due to its extraordinarily high electrical and thermal conductivities, great mechanical strength, large specific surface area, and potentially low manufacturing cost. The excellent properties of high specific surface area $\left(2675 \mathrm{~m}^{2} \mathrm{~g}^{-1}\right)$ and high electrical conductivity have made it a suitable material for supercapacitor applications. Use of thermally exfoliated GN nanosheets as supercapacitor electrode materials has been reported to give a maximum specific capacitance of $117 \mathrm{~F} \mathrm{~g}^{-1}$ in aqueous $\mathrm{H}_{2} \mathrm{SO}_{4}$ electrolyte. For supercapacitors made of chemically modified GN, a specific capacitance of $135 \mathrm{~F} \mathrm{~g}^{-1}$ in 
aqueous $\mathrm{KOH}$ electrolyte has been reported. However, when drying GN during electrode preparation process, the irreversible agglomeration and restacking of GN due to van der Waals interactions to form graphite becomes a major problem for GN based supercapacitors. The agglomeration adversely affects supercapacitor performance by preventing electrolyte from penetrating into the layers. This problem can be avoided by the introduction of spacers into the GN layers. CNTs, metal oxides and conducting polymers can be used as the spacers. Spacers can ensure high electrochemical utilization of GN layers; in addition, electroactive spacers also contribute to the total capacitance. In this section, recent developments on GNbased nanocomposite materials for supercapacitor applications will be reviewed.

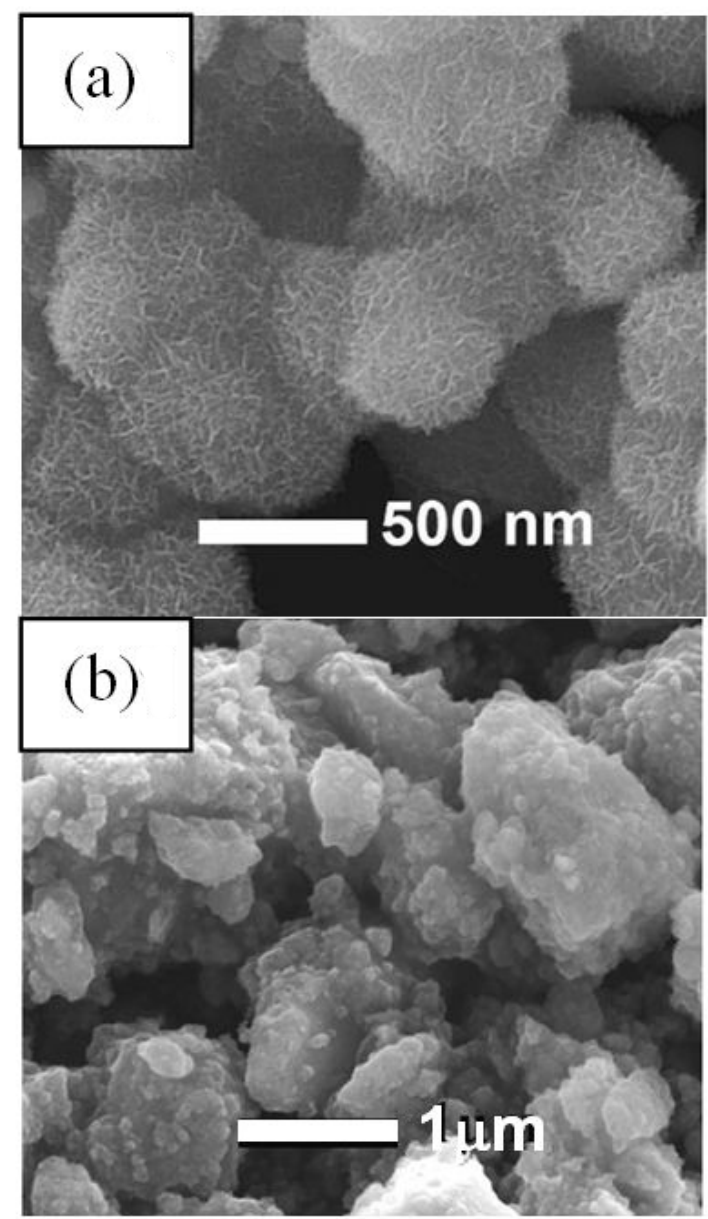

Figure 11. SEM micro-images of MnO2-PANI nanocomposites synthesized by (a) interfacial synthesis and (b) chemical co-precipitation (from ref. 30). 


\subsubsection{Graphene and metal oxide nancomposites}

Metal oxides such as $\mathrm{CeO}_{2}, \mathrm{RuO}_{2}, \mathrm{~V}_{2} \mathrm{O}_{5}$ and $\mathrm{SnO}_{2}$ were used to composite with $\mathrm{GN}$ to form advance nanocomposite for supercapacitor applications. Synergistic effect contributed from GN and metal oxide due to improved conductivity of metal oxide and better utilization of GN is expected to contribute to enhance the pseudocapacitance. Jaidev et al. [36] prepared $\mathrm{RuO}_{2} \bullet \mathrm{xH}_{2} \mathrm{O}-\mathrm{GN}$ nanocomposite by hydrothermal treatment of GN nanosheets, synthesized via exfoliation of graphite oxide in hydrogen environment, with ruthenium chloride in a Teflon-lined autoclave. A symmetrical supercapacitor was fabricated using electrodes prepared by mixing the as-prepared $\mathrm{RuO}_{2} \mathrm{xH}_{2} \mathrm{O}-\mathrm{GN}$, activated carbon and Nafion (binder) on conducting carbon fabric. The hybrid nanocomposite shows a maximum specific capacitance of $154 \mathrm{~F}$ $\mathrm{g}^{-1}$ and energy density of about $11 \mathrm{Wh} \mathrm{kg}^{-1}$ at a specific discharge current of $1 \mathrm{~A} \mathrm{~g}^{-1}$ (20 wt.\% Ru loading). The composite also shows a maximum power density of $5 \mathrm{~kW} \mathrm{~kg}^{-1}$ and coulombic efficiency of $97 \%$ for a specific discharge current of $10 \mathrm{~A} \mathrm{~g}^{-1}$. $\mathrm{CeO}_{2}$ was also deposited onto the 3D GN by chemical precipitation of 3D GN materials contained $\mathrm{Ce}\left(\mathrm{NO}_{3}\right)_{3}$ solution with $\mathrm{KOH}$ [37]. $\mathrm{CeO}_{2}-\mathrm{GN}$ nanocomposite gave high specific capacitance $\left(208 \mathrm{~F} \mathrm{~g}^{-1}\right.$ or $\left.652 \mathrm{mF} \mathrm{cm}^{-2}\right)$ and long cycle life although the specific surface area of the composite decreases as compared with pure GN. Bonso et al. synthesized $\mathrm{GN}-\mathrm{V}_{2} \mathrm{O}_{5}$ nanocomposite by mixing $\mathrm{V}_{2} \mathrm{O}_{5}$ sol with the GN/ethanol dispersion and stirred for many days [38]. The thus-prepared $\mathrm{GN}_{-} \mathrm{V}_{2} \mathrm{O}_{5}$ composite electrode achieved specific capacitance value of $226 \mathrm{~F} \mathrm{~g}^{-1}$ in $1 \mathrm{M}$ LiTFSI in acetonitrile. In contrast, the specific capacitance of just $\mathrm{V}_{2} \mathrm{O}_{5}$ was $70 \mathrm{~F} \mathrm{~g}^{-1}$ and just $\mathrm{GN}$ was $42.5 \mathrm{~F} \mathrm{~g}^{-1}$, demonstrating the synergistic effect of combining the two materials.

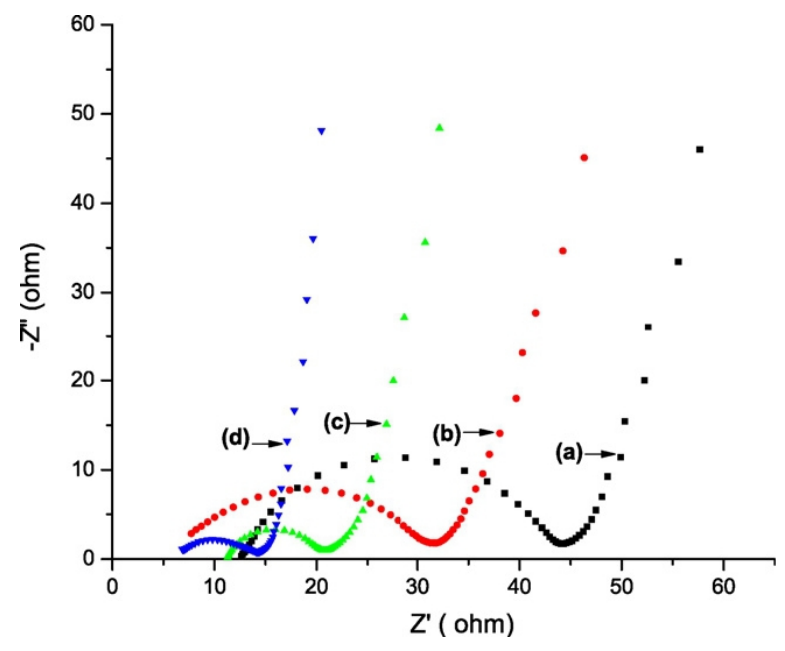

Figure 12. Typical Nyquist impedance plot at open circuit potential (OCP) over a frequency range of $100 \mathrm{kHz}-10 \mathrm{mHz}$ with a potential amplitude of $5 \mathrm{mV}$ for (a) PEDOT-Aq, (b) PEDOT-Org, (c) nano- $\mathrm{NiFe}_{2} \mathrm{O}_{4}$ and (d) PEDOT- $\mathrm{NiFe}_{2} \mathrm{O}_{4}$ Composite electrodes (from ref. 32) 


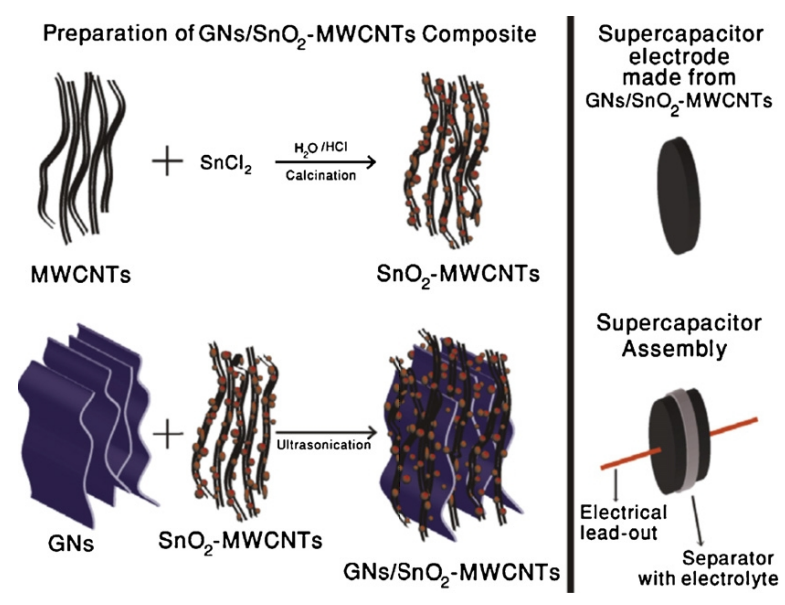

Figure 13. Schematic of preparation of supercapacitor electrode material (from ref. 39).

Transition metal oxide nanoparticles loaded CNTs has been demonstrated as an excellent electroactive material for supercapacitor applications. It is expected that a nanocomposite obtained by dispersion of metal oxide nanoparticles loaded multi-walled CNTs (MWCNTs) into GN can be a good electroactive materials for supercapacitors particularly to increase their cycling stability due to more open structures. Rakhi et al. [39] has prepared the GN$\mathrm{SnO}_{2} / \mathrm{CNTs}$ nanocomposite by ultrasonically mixing of chemically functionalized GN and $\mathrm{SnO}_{2}-\mathrm{CNT}$. The $\mathrm{SnO}_{2} / \mathrm{CNTs}$ was first prepared by chemical precipitation of $\mathrm{SnO}_{2}$ from $\mathrm{SnCl}_{2}$ solution containing functionalized multi-walled CNTs. The $\mathrm{SnO}_{2} / \mathrm{CNTs}$ precipitate was filtrated, washed, dried and calcined. It was then mixed with functionalized GN by ultrasonication to obtain a homogeneous $\mathrm{GN}-\mathrm{SnO}_{2} / \mathrm{CNTs}$ suspension. Finally, the solid was filtered, washed and dried in a vacuum. To produce chemically functionalized graphene, GN was dispersed in concentrated nitric and sulphuric acid mixture. The process for preparation of $\mathrm{GN}-\mathrm{SnO}_{2} / \mathrm{CNTs}$ composite is illustrated in Figure 13. The TEM images of multiwall CNTs and $\mathrm{SnO}_{2} / \mathrm{CNTs}$ are shown in Figure 14 (a) and (b) respectively. Multi-walled CNTs have an average inner diameter of $10 \mathrm{~nm}$, an outer diameter of $30 \mathrm{~nm}$ and an average length in the range of $10-30 \mu \mathrm{m}$. Figure 14(b) suggests an uniform distribution of $\mathrm{SnO}_{2}$ nanoparticles over the surface of multi-walled CNTs. High resolution TEM image of $\mathrm{SnO}_{2} / \mathrm{CNTs}$ (inset of Figure 14(b)) reveals that the $\mathrm{SnO}_{2}$ nanoparticles are highly crystalline in nature with an average particle size of 4-6 nm. TEM images of large area GN and $\mathrm{GN}-\mathrm{SnO}_{2} / \mathrm{CNTs}^{-}$composite are shown in Figure $14(\mathrm{c})$ and (d) respectively. $\mathrm{SnO}_{2} / \mathrm{CNT}$ are seen to occupy the surface of GN. Symmetric supercapacitor devices were fabricated by the authors using GN and $\mathrm{GN}-\mathrm{SnO}_{2} / \mathrm{CNTs}$ composite electrodes. The latter gave remarkable results with a maximum specific capacitance of $224 \mathrm{~F} \mathrm{~g}-1$, power density of $17.6 \mathrm{~kW} \mathrm{~kg}^{-1}$ and an energy density of 31 $\mathrm{Wh} \mathrm{kg}^{-1}$. The results demonstrated that dispersion of metal oxide loaded multi-walled CNTs improved the capacitance properties of GN. The fabricated supercapacitor device exhibited excellent cycle life with $\sim 81 \%$ of the initial specific capacitance retained after 6000 cycles. 


\subsubsection{Graphene and polymer nancomposites}

Conducting polymers such as PANI and PEDOT were composited with GN to improve the electrochemical performance of GN for supercapacitor applications. GN-PANI nanocomposite was chemically synthesized by oxidative polymerization of aniline monomer using ammonium peroxydisulfate $\left.\left[\left(\mathrm{NH}_{4}\right)_{2} \mathrm{~S}_{2} \mathrm{O}_{8}\right)\right]$ as the oxidizing agent in the $\mathrm{GN}$ and aniline mixing solution [40]. The presence of GN in polyaniline shows the penetrating network like structure in GN-PANI nanocomposite film, whereas the GN platelets are making the network structure with polyaniline. The high specific capacitance and good cyclic stability have been achieved using 1:2 aniline to GN ratio by weight. The result of Gómeza et al. [40] has proved that the presence of GN in network of polyaniline changes the composite structure. The supercapacitor fabricated using GN-PANI shows the specific capacitance of $300-500 \mathrm{~F} \mathrm{~g}^{-1}$ at a current density of $0.1 \mathrm{~A} \mathrm{~g}^{-1}$.
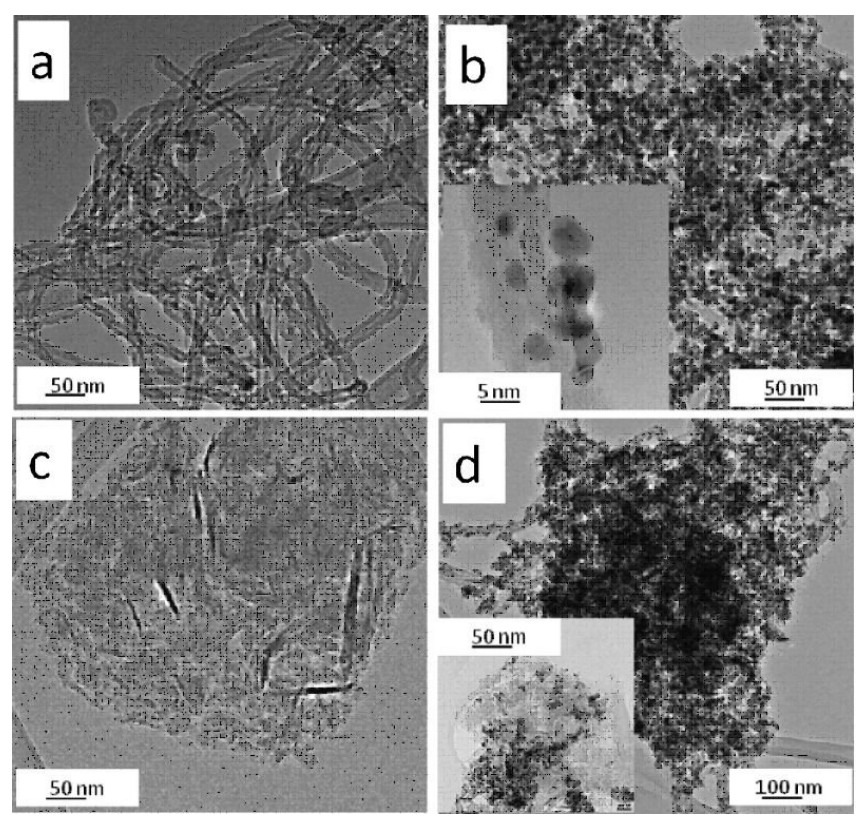

Figure 14. TEM images of (a) MWCNTs, (b) $\mathrm{SnO}_{2}-\mathrm{MWCNTs}$ (inset shows HRTEM image), (c) GNs and (d) GNs/SnO2MWCNTs composite (from ref. 39)

GN-PANI composite film with layered structure was obtained via filtration of an aqueous dispersion consisting of positively charged PANI nanofibres and negative charged chemically converted GN sheets that form a stable composite dispersion via electrostatic interaction with the assistance of ultrasonication [41]. The conductivity of GN-PANI film was one order higher than that of pure PANI nanofibres film. The symmetric supercapacitor device using GN-PANI films exhibited a high capacitance of $210 \mathrm{~F} \mathrm{~g}^{-1}$ at $0.3 \mathrm{~A} \mathrm{~g} \mathrm{~g}^{-1}$, and this capacitance can be maintained for about $94 \%\left(197 \mathrm{~F} \mathrm{~g}^{-1}\right)$ as the discharging current density was increased 
from 0.3 to $3 \mathrm{~A} \mathrm{~g}^{-1}$. Due to the synergic effect of both components, the performance of GNPANI based capacitor is much higher than those of the supercapacitors based on pure chemically converted GN or PANI-nanofibre films. The GN-PANI film has a layered structure as shown in its cross-section scanning electron micrograph (SEM) of Figure 15 (a), which is probably caused by the flow assembly effect of GN sheets during filtration. The magnified SEM image (Figure 15(b)) reveals that PANI nanofibres are sandwiched between chemically converted GN layers. The interspaces between the chemically converted GN layers are in the range of 10-200 nm. This morphology endows GN-PANI film with additional specific surface area comparing with that of the compact GN film prepared under the same conditions (Figure 15c). Filtrating of the dispersion PANI-nanofibres also produced a porous film as shown in Figure 15d, however, the mechanical property of this film is poor and it usually breaks into small pieces after drying.

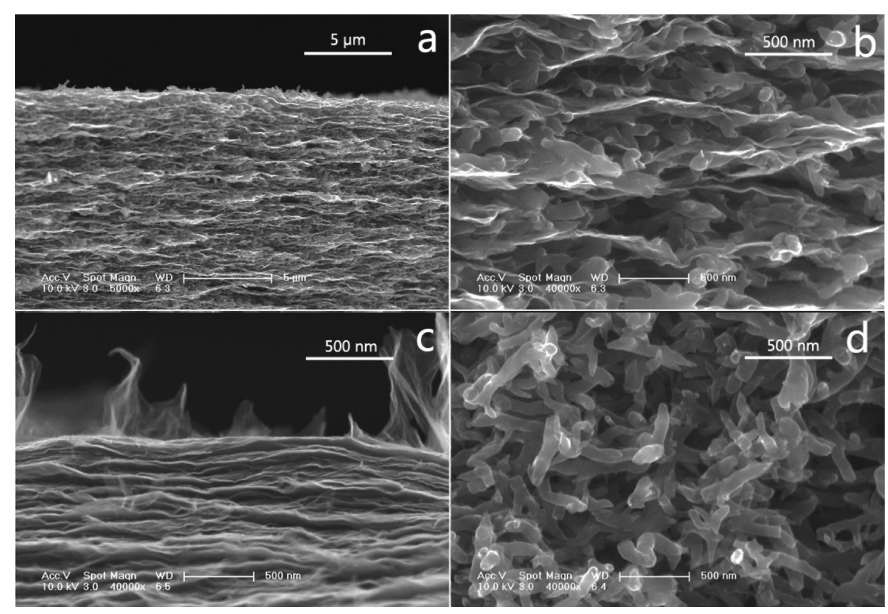

Figure 15. Cross-section SEM images of GN-PANI (a, b), pure chemically converted GN (c), and PANI nanofibre (d) films prepared by vacuum filtration (from ref. 41)

GN was also composited with PEDOT by F. Alvi et al. [42], by chemically oxidative polymerization of ethylene dioxythiophene (EDOT) using ammonium peroxydisulfate $\left.\left[\left(\mathrm{NH}_{4}\right)_{2} \mathrm{~S}_{2} \mathrm{O}_{8}\right)\right]$ and $\mathrm{FeCl}_{3}$ as oxidizing agents. In the solution of EDOT monomer, GN was added into it at EDOT to GN ratio of 1:1. The GN-PEDOT nanocomposite dramatically improves the electrochemical performance comparing to PEDOT based supercapacitors. The GN-PEDOT has also provided faster electrochemical reaction with an average capacity of $350 \mathrm{~F} \mathrm{~g}^{-1}$. All the above results been demonstrated that the improvement in supercapacitor performance of GN based electroactive material can be achieved by compositing with metal oxides or conducting polymers. GN in those nanocomposites can act as nanoscale supports for dispersing metal oxides or conducting polymers to increase their surface area. GN can also provide the electronic conductive channels for metal oxides and conducting polymers. In addition, GN nanosheets can restrict the mechanical deformation of the polymers during 
the redox process due to its unique structural and mechanical properties. Graphene-based nanocomposites are expected to have great future for their application in supercapacitors.

\section{Conclusion and future directions}

Nanocomposite electroactive materials that have been developed so far have demonstrated huge potential for supercapacitor applications. Different types of nanocomposite electroactive materials, such as mixed metal oxides, polymers mixed with metal oxides, carbon nanotubes mixed with polymers, or metal oxides, and graphene mixed with metal oxides or polymers, can be fabricated by various processes such as solid state reactions, mechanical mixing, chemical coprecipitation, electrochemical anodic deposition, sol-gel, in situ polymerization and other wetchemical synthesis. It has been shown that significant improvement in term of specific surface area, electrical and ionic conductivities; specific capacitance, cyclic stability, and energy and power density, of supercapacitors can be achieved by using nanocomposite electroactive materials. This can be attributed to the complementary and synergy behaviours of the consisting material components, the unique interface characteristics and the significant increase in surface areas, as well as nano-scale dimensional effects. Electrochemical double layer capacitors using carbon based electroactive materials and pseudocapacitors using metal oxide or conducting polymers as electroactive materials are the two types of most common supercapacitor structures. Compared with the electrochemical double layer capacitors, pseudocapacitors present a number of advantages such as high energy density and low materials cost, but suffer from poor cyclic stability and lower power density. Asymmetric supercapacitors using one electrode of pseudocapacitive materials and the other carbon based double capacitive materials are of great interest and are the current research focus. Nanocomposite pseudocapactive materials have great potential for asymmetric supercapacitor applications. The key issue is to fully utilizenanocomposites' excellent intrinsically properties, especially their high surface area and high conductivity, and to improve the synergistic effect of different electroactive components.

Although nanocomposite films have demonstrated their great potential for supercapacitor applications, several challenges still remain. Synthesis of nanocomposite electroactive materials with precisely controlling their chemical composition ratio, micro/nanostructures, phases, surface area and interfacial characteristics is still challenging. Depending on the preparation technique and process parameters, the property and behaviours of the nanocomposite electroactive materials can vary significantly; therefore, the ability to reproducibly synthesize nanocomposite materials with consistent properties is very important for their wide uses in supercapacitors. Degradation of the nanocomposite electroactive materials stemming from aggregation of the nano-scale components due to the relatively strong forces between them, micro/nanostructure changes due to charging-discharging cycling and materials contaminations due to impurity introduced for original reactants or during synthesis processes etc. has to be resolved before their large-scale adoption by the industry. Most importantly, the costs of materials and their synthesis processes have to be reduced significantly. With the increase in interest and intensive research and development, it is ex- 
pected that, nanocomposite electroactive materials will have a promising future and will bring a huge change to the energy storage industries.

\section{Acknowledgements}

The author would like to thank Transport Canada and National Research Council of Canada (NRC) for supporting the publication of this chapter. The author is also indebted to his NRC colleagues: Dr. Sylvain Pelletier and Ms. Nathalie Legros for their initiation of supercapacitor research at NRC, and Dr. Alexis Laforgue, Dr. Lucie Robitaille, Dr. Yves Grincourt, Dr. Lei Zhang, and Dr. Jiujun Zhang for their collaboration in the supercapacitor research project. He would also like to thanks his research team members: Mr. Brian Gibson, Mr. Marco Zeman, Mr. Robinet Romain, Mr. Benjamin Tailpied, and Ms. Gaëlle LEDUC for their dedication to supercapacitor research. Thank is also given to Ms. Catherine Yang for her editorial review on this chapter.

\section{Author details}

Dongfang Yang*

Address all correspondence to: Email: dongfang.yang@nrc.gc.ca

National Research Council Canada, 800 Collip Circle, London, Ontario,, Canada

\section{References}

[1] Jiang, H., Li, C., Sun, T., \& Ma, J. (2012). High-performance supercapacitor material based on $\mathrm{Ni}(\mathrm{OH})_{2}$ nanowire- $\mathrm{MnO}_{2}$ nanoflakes core-shell nanostructures. Chem. Commun., 48, 2606-2608.

[2] Kim, H., \& Popov, N. (2003). Synthesis and Characterization of MnO2Based Mixed Oxides as Supercapacitors. Journal of the Electrochemical Society, 150(3), D56-D62.

[3] Lee, M. T., Chang, J. K., Hsieh, Y. T., \& Tsai, W. T. (2008). Annealed Mn-Fe binary oxides for supercapacitor applications. Journal of Power Sources, 185-1550.

[4] Ye, Z. G., Zhou, X. L., Meng, H. M., Hua, X. Z., Dong, Y. H., \& Zou, A. H. (2011). The Electrochemical Characterization of Electrochemically Synthesized $\mathrm{MnO} 2$ based Mixed Oxides for Supercapacitor Applications. Advanced Materials Research, 287(290), 1290-1298.

[5] Yang. (2012). Pulsed laser deposition of cobalt-doped manganese oxide thin films for supercapacitor applications. Journal of Power Sources, 198-416. 
[6] Yang, D. (2011). Pulsed laser deposition of manganese oxide thin films for supercapacitor Applications. Journal of Power Sources, 196-8843.

[7] Zhong, J. H., Wang, A. L., Li, G. R., Wang, J. W., Ou, Y. N., \& Tong, Y. X. (2012). $\mathrm{Co}_{3} \mathrm{O}_{4} / \mathrm{Ni}(\mathrm{OH})_{2}$ composite mesoporous nanosheet networks as a promising electrode for supercapacitor applications. J. Mater. Chem., 22-5656.

[8] Yang, Y., Kim, D., Yang, M., \& Schmuki, P. Vertically aligned mixed 2O5-TiO2 nanotube arrays for supercapacitor applications. 2011, Chem. Commun., 47, 7746-7748.

[9] Jayalakshmi, M., Venugopal, N., Phani, Raja. K., \& Mohan, Rao. (2006). Nano SnO2 $\mathrm{Al}_{2} \mathrm{O}_{3}$ mixed oxide and $\mathrm{SnO}_{2}-\mathrm{Al}_{2} \mathrm{O}_{3}$-carbon composite oxides as new and novel electrodes for supercapacitor applications. Journal of Power Sources, 158, 1538-1543.

[10] Wang, C., Zhang, X., Zhang, D., \& Yao, C. (2012). Facile and low-cost fabrication of nanostructured $\mathrm{NiCo} 2 \mathrm{O} 4$ spinel with high specific capacitance and excellent cycle stability. Electrochimica Acta, 63-220.

[11] Chang, S. K., Lee, K. T., Zainal, Z., Tan, K. B., Yusof, N. A., Yusoff, W., Lee, J. F., \& $\mathrm{Wu}$, N. L. (2012). Structural and electrochemical properties of manganese substituted nickel cobaltite for supercapacitor application. Electrochimica Acta, 67.

[12] An, K. H., Jeon, K. K., Heo, J. K., Lim, S. C., Bae, D. J., \& Lee, Y. (2002). High-Capacitance Supercapacitor Using a Nanocomposite Electrode of Single-Walled Carbon Nanotube and Polypyrrole. Journal of The Electrochemical Society, 149(8), A1058-A1062.

[13] Deng, M., Yang, B., \& Hu, Y. (2005). Polyaniline deposition to enhance the specific capacitance of carbon nanotubes for supercapacitors. Journal of Materials Science Letters.

[14] Tadai, K., \& Mitani, T. (2005). Highly dispersed ruthenium oxide nanoparticles on carboxylated carbon nanotubes for supercapacitor electrode materials. J. Mater. Chem., 15-4914.

[15] Kim, Y., \& Mitani, T. (2006). Oxidation treatment of carbon nanotubes: An essential process in nanocomposite with $\mathrm{RuO}_{2}$ for supercapacitor electrode materials. Appl. Phys. Lett., 89, 033107.

[16] Hsieh, T. F., Chuang, C. C., Chen, W. J., Huang, J. H., Chen, W. T., \& Shu, C. M. (2012). Hydrous ruthenium dioxide/multi-walled carbon-nanotube/titanium electrodes for supercapacitors. Oxidation treatment of carbon nanotubes:. An essential process in nanocomposite with RuO2 for supercapacitor electrode materials, 50, 1740-1747.

[17] Lee, J. K., Pathan, H. M., Jung, K. D., \& Joo, O. S. (2006). Electrochemical capacitance of nanocomposite films formed by loading carbon nanotubes with ruthenium oxide. Journal of Power Sources, 159-1527.

[18] Kim, B. C., Wallace, G. G., Yoon, Y. I., Ko, J. M., \& Too, C. (2009). Capacitive properties of $\mathrm{RuO}_{2}$ and $\mathrm{Ru}-\mathrm{Co}$ mixed oxide deposited on single-walled carbon nanotubes for high-performance supercapacitors. Synthetic Metals, 159-1389. 
[19] Chen, Y. M., Cai, J. H., Huang, Y. S., Lee, K. Y., \& Tsai, D. (2011). Preparation and characterization of iridium dioxide-carbon nanotube nanocomposites for supercapacitors. Nanotechnology, 22(115706), 7.

[20] Lee, C. Y., Tsai, H. M., Chuang, H. J., Li, S., Lin Yi, P., \& Tseng, T. (2005). Characteristics and Electrochemical Performance of Supercapacitors with Manganese OxideCarbon Nanotube Nanocomposite Electrodes. Journal of The Electrochemical Society, 152(4), 716A-A720.

[21] Lee, J. Y., Liang, K., An, K. H., \& Lee, Y. (2005). Nickel oxide/carbon nanotubes nanocomposite for electrochemical capacitance. Synthetic Metals, 150-153.

[22] Liu, M. Y., Hsieh, T. F., Hsieh, C. J., Chuang, C. C., \& Shu, C. M. (2011). Performance of vanadium oxide on multi-walled carbon nanotubes/titanium electrode for supercapacitor application. Advanced Materials Research, 311-313, 414-418.

[23] Jayalakshmi, M., Mohan, Rao. M., Venugopal, N., \& Kim, K. B. (2007). Hydrothermal synthesis of $\mathrm{SnO}_{2}$-2O5 mixed oxide and electrochemical screening of carbon nanotubes (CNT), $\mathrm{V}_{2} \mathrm{O}_{5}, \mathrm{~V}_{2} \mathrm{O}_{5}-\mathrm{CNT}$, and $\mathrm{SnO}_{2}-\mathrm{V}_{2} \mathrm{O}_{5}-\mathrm{CNT}$ electrodes for supercapacitor applications. Journal of Power Sources, 166, 578-583.

[24] Lee, B. J., Sivakkumar, S. R., Ko, J. M., Kim, J. H., Jo, S. M., \& Kim, D. (2007). Carbon nanofibre/hydrous $\mathrm{RuO}_{2}$ nanocomposite electrodes for supercapacitors. Journal of Power Sources, 168-546.

[25] Cormier, Z. R., Andreas, H. A., \& Zhang, P. (115). Temperature-Dependent Structure and Electrochemical Behaviour of $\mathrm{RuO}_{2} /$ Carbon Nanocomposites. J. Phys. Chem, 115, 19117-19128.

[26] Selvakumar, M., Bhat, D. K., Aggarwal, A. M., Iyer, S. P., \& Sravani, B. (2010). Physica, 405-2286.

[27] Tao, T., Chen, Q., Hu, H., \& Chen, Y. (2012). $\mathrm{MoO}_{3}$ nanoparticles distributed uniformly in carbon matrix for supercapacitor applications. Materials Letters, 66-102.

[28] Chen, L., Sun, L., Luan, F., Liang, Y., Li, Y., Liu, X., \& , X. (2010). Synthesis and pseudocapacitive studies of composite films of polyaniline and manganese oxide nanoparticles. Journal of Power Sources, 195-3742.

[29] Hu, Z., Xie, Y., Wang, Y., Mo, L., Yang, Y., \& Zhang, Z. (2010). Synthesis and pseudocapacitive studies of composite films of polyaniline and manganese oxide nanoparticles. Journal of Power Sources, 195-3742.

[30] Wang, J., Yang, Y., \& Huang, Z. (2012). . Interfacial synthesis of mesoporous MnO2/ polyaniline hollow spheres and their application in electrochemical capacitors. Journal of Power Sources, 204-236.

[31] Saranya, S., \& Selvan, R. K. (2012). Synthesis and characterization of polyaniline/ $\mathrm{MnWO}_{4}$ nanocomposites as electrodes for pseudocapacitors. Applied Surface Science, 258-4881. 
[32] Sen, P. (2010). Electrochemical performances of poly 3 4ethylenedioxythiophene)$\mathrm{NiFe}_{2} \mathrm{O}_{4}$ nanocomposite as electrode for supercapacitor. Electrochimica Acta, 55, 4677-4684.

[33] Murugan, A. V., Viswanath, A. K., \& Gopinath, C. S. (2006). Highly efficient organicinorganic poly (3 4-ethylenedioxythiophene)-molybdenum trioxide nanocomposite electrodes for electrochemical supercapacitor. J. Appl. Phys., 100(074319), 1-5.

[34] Dong, Z. H., Wei, Y. L., Shi, W., \& Zhang, G. (2011). Characterisation of doped polypyrrole/manganese oxide nanocomposite for supercapacitor electrodes. Materials Chemistry and Physics, 131-529.

[35] Yang, X., Wang, G., Wang, R., \& Li, X. (2010). A novel layered manganese oxide/poly (aniline-co-o-anisidine) nanocomposite and its application for electrochemical supercapacitor. Electrochimica Acta, 55-5414.

[36] Jaidev, Jafri. R. I. (2011). Ramaprabhu S. Hydrothermal synthesis of $\mathrm{RuO}_{2} \cdot \mathrm{xH}_{2} \mathrm{O}$ / graphene hybrid nanocomposite for supercapacitor application. International Conference on Nanoscience,. Technology and Societal Implications, 8-10.

[37] Wang, Y., Guo, C. X., Liu, J., Chen, T., Yang, H., \& Li, C. (2011). $\mathrm{CeO}_{2}$ nanoparticles/ graphene nanocomposite-based high performance supercapacitor. Dalton Trans., 40(6388).

[38] Bonso, J. S., Rahy, A., Perera, S. D., Nour, N., Seitz, O., Chabal, Y. J., Balkus Jr, K. J., Ferraris, J. P., \& Yang, D. (2012). Exfoliated graphite nanoplatelets-2O5 nanotube composite electrodes for supercapacitors. Journal of Power Sources, 203, 227-232.

[39] Rakhi, R. B., \& Alshareef, H. N. (2011). Enhancement of the energy storage properties of supercapacitors using graphene nanosheets dispersed with metal oxide-loaded carbon nanotubes. Journal of Power Sources, 196-8858.

[40] Gómeza, H., Ramb, M. K., Alvia, F., Villalba, P., \& Stefanakos, E. (2011). Grapheneconducting polymer nanocomposite as novel electrode for Supercapacitors. Journal of Power Sources, 196-4102.

[41] Wu, Q., Xu, Y., Yao, Z., \& Liu, A. (2010). Supercapacitors Based on Flexible Graphene/Polyaniline Nanofiber Composite Films. ACS. Nano, 4(4), 1963-1970.

[42] Stefanakos, E., Goswami, Y., \& Kumar, A. (2011). Graphene-polyethylenedioxythiophene conducting polymer nanocomposite based supercapacitor. Electrochimica Acta, 56-9406. 